\title{
Designing a performance measurement system for supply chain using balanced scorecard, path analysis, cooperative game theory and evolutionary game theory: A Case Study
}

\author{
Seyed Hootan Eskafi ${ }^{a^{*}}$, Emad Roghanian ${ }^{a}$ and Meisam Jafari-Eskandari ${ }^{\mathrm{b}}$
}

${ }^{a}$ Department of Industrial Engineering, Khaje Nasir university of Technology, Tehran, Iran

${ }^{b}$ Department of Industrial Engineering, Payame Noor university, Tehran, Iran

\begin{abstract}
A B S T R A C T
In recent years, supply chain management is known as the key factor for achieving competitive advantage. Better customer service, revenue improvement and cost reduction are the results of this philosophy. Organizations can manage the performance of their firms by appropriate goal setting, identifying criteria and continuous performance measurement, which creates a good view for the business circumstances. Developing and defining appropriate indicators at different levels of chain is necessary for implementing a performance measurement system. In this study, we propose a new method to determine the measurement indicators and strategies of the company in term of balanced scorecard. The study is a combination of balanced scorecard, path analysis, evolutionary game theory and cooperative game theory for strategic planning. The study offers an appropriate program for future activities of organizations and determines the present status of the firm. The implementation of the proposed method is introduced for a food producer and the results are analyzed.
\end{abstract}

\section{Introduction}

A supply chain is one of the most essential parts of business management for designing services from suppliers to customers. In supply chains with multiple vendors, manufacturers, distributors and retailers, performance measurement is difficult due to the challenges involved in the attribution of performance results to any other element of the chain. Performance measurement in supply chains becomes more difficult for additional reasons, especially since the analysis consists of numerous tiers within the chain itself. Performance measurement is important for managing the supply chain and increasing its effectiveness and efficiency. Decision-makers in supply chains usually focus on defining indices, which are quantitative. The primary purpose of this paper is to use a new method for performance measurement by combining balanced scorecard, path analysis, cooperative game theory and evolutionary game theory through an operational management perspective to demonstrate how these perspectives complement each other to increase efficiency. The study also suggests a strategy in supply chain focusing on a 12 period of six months project to develop performance measurement systems in the TAYHOO Company, which is the production and distribution factory in food industries.

* Corresponding author. Tel:+989359812568

E-mail: hootaneskafi@gmail.com (S. H. Eskafi)

(C) 2014 Growing Science Ltd. All rights reserved. doi: 10.5267/j.ijiec.2014.12.003 
The rest of this paper is organized as follows: A literature review of the performance measurement in the supply chain, the balanced scorecard, path analysis, cooperative game theory and evolutionary game theory are first presented. Next, the proposed framework for the analysis of supply chain performance management and a systematic approach for assigning priorities to different perspectives, based on balanced scorecard (BSC) are given. The study discusses an illustrative application of the proposed methodology by applying it for a case study and the results are analyzed. Upon conclusion we shall discuss the findings and suggest implications that this study has on measuring performance of the chain supply.

\section{Literature review}

This section reviews the literature on BSC systems, path analysis, cooperative game theory and one of the important concepts of this type of games called shapely value. We also focus on a four-person game based on BSC that complete our method.

\subsection{Balanced Scorecard}

Kaplan and Norton $(1992,2004)$ are believed to be the first who introduced balanced scorecard (BSC). BSC comprises of four perspectives of learning and growth, internal processes, customers, and finance. BSC seeks to offer managers a system that would help them turn strategy into action. This system arranges the vision of the organization into action (Kaplan \& Norton, 2006). Presently, a large number of organizations are currently successfully using BSC. According to Koning (2004) $60 \%$ of the 500 largest organizations in Fortune use BSC. Gumbus (2005) also mentions that in 2005, 64\% of American Companies use BSC for performance evaluation. There is a considerable evidence that organizations are increasingly adopting BSC in their strategic processes. The BSC has many benefits for organization some of which include:

1. Only a few measures or performance indicators need to be checked at any one time (Neely, 1998).

2. Serving as a bridge between different fields (financial and nonfinancial fields) (Kaplan \& Norton, 1996).

3. Drawing on causal loop diagrams for improving strategic plans (Kaplan \& Norton, 1996; Li et al., 2009).

4. Improving the management of information in organizations (Huang, 2009; Bobillo et al., 2009).

Fig. 1 shows a scheme of the BSC model originally proposed by Kaplan and Norton in 1996. The BSC has been widely investigated in literature. Yang and Tung (2006) used BSC to evaluate the performance of some hospitals. Also this method have used for the evaluation of power generation from fossil fuels by Razmi et al. (2008). Cebeci (2009) used this system to help implement ERP systems in textile industry in Taiwan. Lee and colleagues (2008) used the BSC approach to evaluate IT department of manufactured companies in Taiwan. Kanji (2002) used BSC for assessing business models. Bhagwat and Sharma (2007) with a review of previous studies assessing the performance of the supply chain, including the papers of Gunasekaran et al. (2004), began to collect important criteria in evaluating supply chain performance. First, they derived the criteria into two categories of financial and non-financial. In this paper, after reviewing the literature, a general framework of indicators was provided to assess overall supply chain performance in terms of four criteria BSC. After presenting the general framework, these criteria have been measured in three small and medium enterprises in India.

The BSC has been widely investigated in literature (Chia et al., 2009), but little attention has been paid by researchers to its adoption in the food industry: among these, it is possible to cite Cardemil-Katuranic and Shadbolt (2006), which adopted a case study methodology to research how an agricultural (kiwifruit) co-operative in New Zealand could implement a BSC. Bigliardi and Bottani (2010) by using studies of 
Bhagwat and Sharma presented a general framework of the BSC measures for food industries supply chain. We use this framework for our method in this paper.

Balanced Scorecard is defined as a cause and effect model to determine in what indicators the organization is leading or in what indicators the organization has a low performance. Balanced Scorecard assumes that the causal relationships are established among the four perspectives of organizational learning and growth, internal process perspective, customer perspective and the financial perspective (Kaplan \& Norton 1996). These relationships are very important because, unlike traditional methods, they allow managers to measure performance based on non-financial criteria and use them to predict organizational financial performance. On the other hand, each cause and effect relationship requires a time interval between cause and its effect. Thus, it is very difficult to create real relationships between all the considered aspects. Different methods have been used for establishing this relationship, including the use of key performance indicator and mathematical modelling. Here, the use of multivariate analysis methods such as path analysis can be a more reliable technique.

\subsection{Path analysis}

Path analysis is a multivariate analysis method that are used for reviewing a set of relationships that form the linear causal models shown. This method was presented for the first time by Sewel Right to explain casual relationships of genetic population. He used this model to study the direct and indirect effects of cause variables to effect variables. Advantages of path analysis compared with other methods such as regression, is: Utility of integrated models of coefficients test rather individually coefficient test, ability of test models with multiple correlation between coefficients.

Huang et al. (2007) used the system for evaluating effective communication in the field of biomedical engineering. Kanji and e Sá (2002) used path analysis for business scorecard analysis that presented by Kanji. Their method is unable to determine the inter-relationships between each of the existing framework because they had used cross partial data. Burney et al. (2009) approach has been used in this study that they used multivariate path analysis method with longitudinal data for analysis of organizational factors. They considered drawing the causal relationships after identifying the effective components. Then, first step relations were examined by assuming statistical under the two-sided t-test. In the end correlation values are desired by regression.

\subsection{Cooperative Game Theory}

Game theory involves a significant part of decision making process under uncertainty conditions. Following its introduction, Game theory was developed by Emily Bourl and Van Noman, although it is not exactly clear that who should be introduced as the founder of the theory. For each game, three factors are necessary:
a) Players
b) Players' strategies that are allowed under rules of the game
c) Utilities or results

In this study, the main approach uses a game with $n$ players and balanced scorecard. Hence, in this section, we try to focus on this type of game and wish to become more familiar with its basic concepts.

Consider a limited number of players $(n)$ shown by a set like $=(1,2,3, \cdots, n)$. Each subset $K \subseteq N$ of this set is called a coalition. $N / K$ is used to display the supplementary set of $K$ in $N$ or $K \subseteq N$ which refers to players who are not in the coalition of $K$. Suppose the coalition with $K$ persons can earn the amount of maximum guaranteed points, $V(K)$. We call $V(K)$ a property function, which is defined in terms of $K \subseteq N$. This function will satisfy the following properties:

1. $V(\varnothing)=0$;

2. For all $K, L \subseteq N$ that $K \geq L \neq \emptyset$, we have $V(K \cup L) \geq V(K)+V(L)$; 
The first property states that the value of an empty coalition is equal to zero. The second one is called additive property and states that the value of a coalition composed of two components is at least equal to their total individual values. Many types of solution concepts have been proposed in the literature for $n$ player cooperative games. One important solution, which is proposed by Shapely in1953, is known as Shapely value. The Shapley value indicates the relative benefit that accrues to each player in a coalition. However, we note that the value applies to transferable utility (games with side paying). Shapely value in non-transferable utility was presented by Shapely in 1969 and extended more by Mashler and Owen in 1992. Suppose that $G(n)$ is the set of all games (all possible property functions) with $n$ players. Shapely value of $\psi$ is a mapping $\psi: G(n) \rightarrow E^{n}$ ( $E^{n}$ is an n-dimensional Euclidean space) that satisfies the following conditions:

1. Symmetry: If players $i, j$ are replaced in a particular game, $V$, then $\psi v_{i}=\psi v_{j}$

$\psi v_{i}$ shows the reward obtained by person $i$ in game $V$ under Shapely value.

2. Performance: $\sum_{i=1}^{n} \psi v_{i}=V(n)$

3. Additive: $\psi(v+h)_{i}=\psi v_{i}+\psi h_{i}$

4. Void player: $\psi v_{\varnothing}=0$

In 1953, Shapely showed that $\psi$ is unique and proved the following theorem:

$$
\psi v_{i}=\frac{1}{n !} \sum_{M}\left[V\left(K_{i} \cup\{i\}\right)-V\left(K_{i}\right)\right]
$$

where $n$ different transforms ( $n$ ! number mode), $M$ changes and $i K$ is a set of players that are placed before $i$ in the sequence of $M$. A proved theorem to calculate the Shapely value when the number of coalition $\mathrm{K}$ is shown with $|\mathrm{K}|$ is as follows:

$$
\psi v_{i}=\sum_{K: i \notin K} \frac{|K| !(n-1-|K|) !}{n !}(V(K \cup\{i\})-V(K)) .
$$

\subsection{Evolutionary Game Theory}

Evolutionary game theory arose as a result of the application of game theory to biological evolutionary contexts and has since been utilized in other areas of social science such as economics and business (Cai \& Kock, 2009). Although evolutionary game theory has provided numerous insights in economics, it has not yet been applied widely in the context of management due to the presence of three main obstacles or limitations of game theory itself described as:

1. The problem of equilibrium selection: Not every game can be seen in the context of a Nash equilibrium which makes solving games more difficult.

2. The problem of hyper-rational agents: The traditional theory of games imposes a very high rationality requirement upon agents. This limitation is not important for all games.

3. The lack of a dynamic theory in the traditional theory of games: Game theory is not dynamic which is very important in modern organizations. Thus evolutionary game theory can be seen, in part, as filling in this gap which exists in traditional game theory.

There are two approaches to evolutionary game theory. The first approach derives from the work of Maynard Smith and Price (1973) and employs the concept of an evolutionarily stable strategy as the principal tool of analysis. The second approach constructs an explicit model of the process by which the frequency of strategies changes in the population and studies properties of the evolutionary dynamics within that model (Adachi et al., 1991; Alexander \& McKenzie, 2000; Gilles, 2009). We use the second approach for this paper. 


\section{Methodology}

This study seeks to contribute by aiming to help us better understanding of characteristics and processes for the design and implementation of enabling performance measurement systems by introducing a new approach in defining perspectives of the balanced scorecard for Food industry supply chain management (SCM). We aim at using a new method for performance measurement by combining balanced scorecard, path analysis, cooperative game theory and evolutionary game theory from an operational management perspective to demonstrate how these perspectives complement each other to increase efficiency and how to determine the best strategic combination. The overall algorithm of this study is shown in Fig. 1. This algorithm consists of four steps. In first step by review on literature of the food supply chains this kind of supply chains are identified and analyzed. Then we have identified strategies related to each of the criteria by using the BSC. In the next step, we determine cause and effect relationships between the strategies defined. For this purpose, the strategic plan will be drawn and relation paths will be defined between the strategies of one criteria and the following criteria. After determining strategic paths, weights for each of the path are obtained by using Shapely Value. The values obtained in this step are considered as input to the Latin square design. In the last step we can choose the best strategies in each criteria by using the evolutionary game theory.

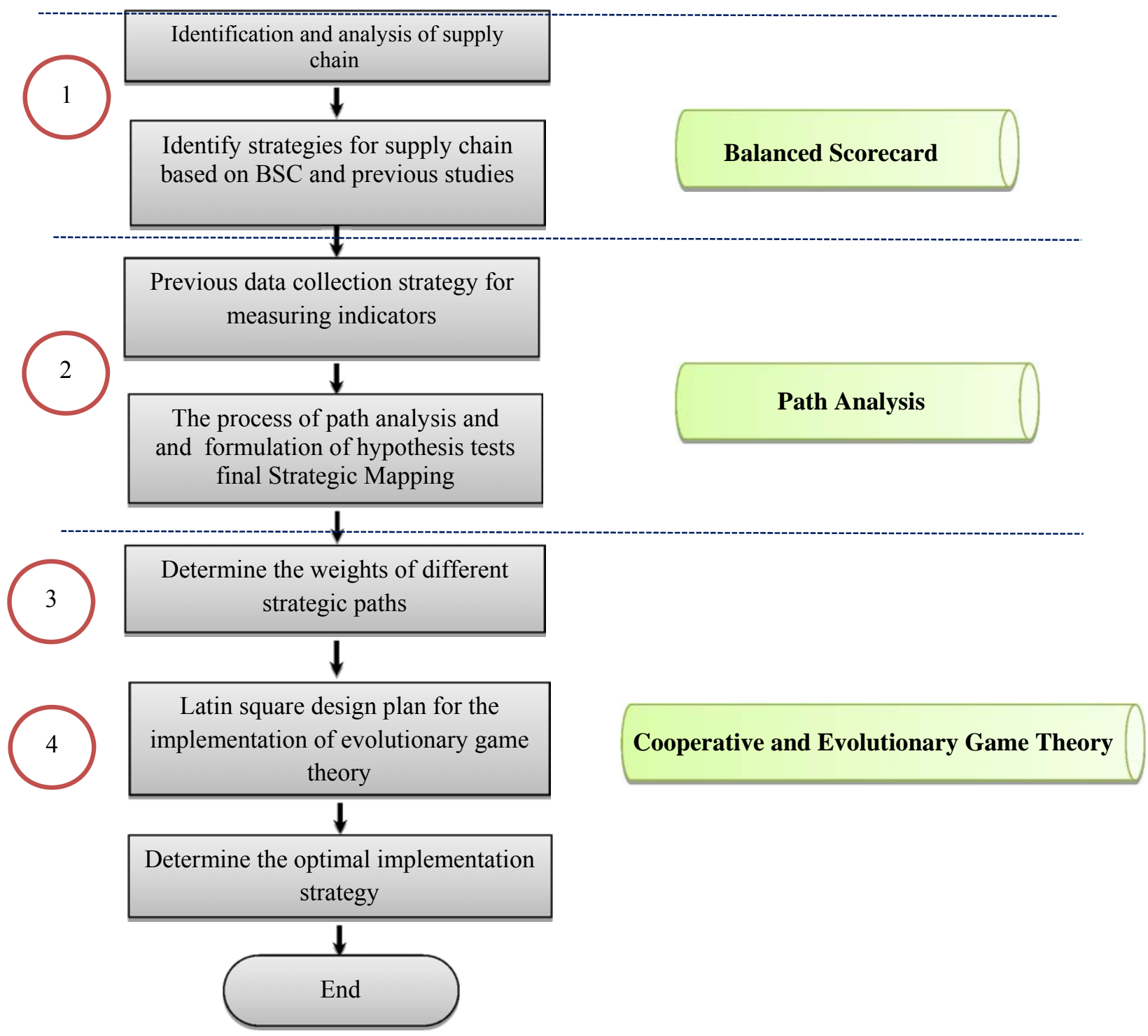

Fig. 1. Methodology of study 
Table 1

Latin square for 4 players' evolutionary game

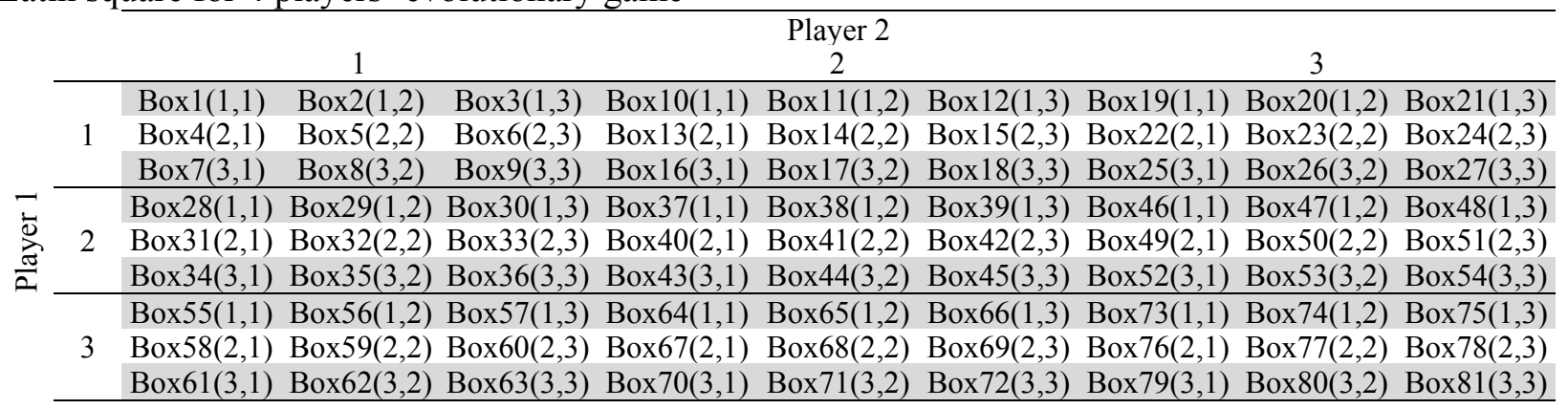

Table 2

Boxes of Latin square for 4 players' evolutionary game

\begin{tabular}{|c|c|c|c|c|c|c|c|c|c|c|}
\hline & & \multicolumn{9}{|c|}{ Player 2} \\
\hline & & \multicolumn{3}{|c|}{1} & \multicolumn{3}{|c|}{2} & \multicolumn{3}{|c|}{3} \\
\hline \multirow{18}{*}{$\begin{array}{l}\overline{\vec{D}} \\
\stackrel{\overrightarrow{\mathrm{d}}}{\underline{a}}\end{array}$} & \multirow{6}{*}{1} & Player 3 (1) & Player 3 (1) & Player 3 (1) & Player 3 (1) & Player 3 (1) & Player 3 (1) & Player 3 (1) & Player 3 (1) & Player 3 (1) \\
\hline & & Player 4 (1) & Player 4 (2) & Player 4 (3) & Player 4 (1) & Player 4 (2) & Player 4 (3) & Player 4 (1) & Player 4 (2) & Player 4 (3) \\
\hline & & Player 3 (2) & Player 3 (2) & Player 3 (2) & Player 3 (2) & Player 3 (2) & Player 3 (2) & Player 3 (2) & Player 3 (2) & Player 3 (2) \\
\hline & & Player 4 (1) & Player 4 (2) & Player 4 (3) & Player 4 (1) & Player 4 (2) & Player 4 (3) & Player 4 (1) & Player 4 (2) & Player 4 (3) \\
\hline & & Player 3 (3) & Player 3 (3) & Player 3 (3) & Player 3 (3) & Player 3 (3) & Player 3 (3) & Player 3 (3) & Player 3 (3) & Player 3 (3) \\
\hline & & Player 4 (1) & Player 4 (2) & Player 4 (3) & Player 4 (1) & Player 4 (2) & Player 4 (3) & Player 4 (1) & Player 4 (2) & Player 4 (3) \\
\hline & \multirow{6}{*}{2} & Player 3 (1) & Player 3 (1) & Player 3 (1) & Player 3 (1) & Player 3 (1) & Player 3 (1) & Player 3 (1) & Player 3 (1) & Player 3 (1) \\
\hline & & Player 4 (1) & Player 4 (2) & Player 4 (3) & Player 4 (1) & Player 4 (2) & Player 4 (3) & Player 4 (1) & Player 4 (2) & Player 4 (3) \\
\hline & & Player 3 (2) & Player 3 (2) & Player 3 (2) & Player 3 (2) & Player 3 (2) & Player 3 (2) & Player 3 (2) & Player 3 (2) & Player 3 (2) \\
\hline & & Player 4 (1) & Player 4 (2) & Player 4 (3) & Player 4 (1) & Player 4 (2) & Player 4 (3) & Player 4 (1) & Player 4 (2) & Player 4 (3) \\
\hline & & Player 3 (3) & Player 3 (3) & Player 3 (3) & Player 3 (3) & Player 3 (3) & Player 3 (3) & Player 3 (3) & Player 3 (3) & Player 3 (3) \\
\hline & & Player 4 (1) & Player 4 (2) & Player 4 (3) & Player 4 (1) & Player 4 (2) & Player 4 (3) & Player 4 (1) & Player 4 (2) & Player 4 (3) \\
\hline & \multirow{6}{*}{3} & Player 3 (1) & Player 3 (1) & Player 3 (1) & Player 3 (1) & Player 3 (1) & Player 3 (1) & Player 3 (1) & Player 3 (1) & Player 3 (1) \\
\hline & & Player 4 (1) & Player 4 (2) & Player 4 (3) & Player 4 (1) & Player 4 (2) & Player 4 (3) & Player 4 (1) & Player 4 (2) & Player 4 (3) \\
\hline & & Player 3 (2) & Player 3 (2) & Player 3 (2) & Player 3 (2) & Player 3 (2) & Player 3 (2) & Player 3 (2) & Player 3 (2) & Player 3 (2) \\
\hline & & Player 4 (1) & Player 4 (2) & Player 4 (3) & Player 4 (1) & Player 4 (2) & Player 4 (3) & Player 4 (1) & Player 4 (2) & Player 4 (3) \\
\hline & & Player 3 (3) & Player 3 (3) & Player 3 (3) & Player 3 (3) & Player 3 (3) & Player 3 (3) & Player 3 (3) & Player 3 (3) & Player 3 (3) \\
\hline & & Player 4 (1) & Player 4 (2) & Player 4 (3) & Player 4 (1) & Player 4 (2) & Player 4 (3) & Player 4 (1) & Player 4 (2) & Player 4 (3) \\
\hline
\end{tabular}

We use a 4 player game in this study. Each player proposed in this study has three strategic options. Each strategy is mutually exclusive, meaning only one can be chosen at any given time. Therefore there will be 81 possibilities of interaction $(3 \times 3 \times 3 \times 3)$. These interactions will be arranged in four distinct boxes of Table 1 and Table 2 . We propose interaction among the different strategic agents of the scorecard as players providing a methodology for collaboration among different players to reduce any inconsistencies and implement a four-person cooperative evolutionary game theory as a new concept in game theory concept. Mathematical equations are used for concepts of the evolutionary game theory. Let $P_{i}$ proportion of population that selected boxed $i^{\text {th }}$. Furthermore, let $W_{j i}$ denote the average fitness of strategy $i^{t h}$ for player $j^{\text {th }}$, respectively, and let $\bar{W}_{i}$ denote the average fitness of the entire population. Furthermore, suppose that each individual in the population has an initial fitness of $F_{j k}$ that explain of value of strategy $k^{\text {th }}$ selected by player $j^{\text {th }}$. Let $\Delta F\left(s_{1}, s_{2}, s_{3}, s_{4}\right)$ denote the change in fitness. The values of $W_{j i}$ and $\bar{W}_{i}$ can be expressed in terms of the population proportions and payoff values as follows:

$$
\begin{gathered}
W_{1}^{1}=F_{1}^{1}+P_{1} \Delta F(1,1,1,1)+P_{2} \Delta F(1,1,1,2)+P_{3} \Delta F(1,1,1,3)+\cdots+P_{25} \Delta F(1,3,3,1) \\
+P_{26} \Delta F(1,3,3,2)+P_{27} \Delta F(1,3,3,3)
\end{gathered}
$$

The above equation has been chosen for the first player when the first strategy is achieved. Following this equation, the first player with the first strategy is assumed constant. All other compounds are identified similar to the above equation. Accordingly, the next equations are:

$$
\begin{gathered}
W_{2}^{1}=F_{2}^{1}+P_{28} \Delta F(2,1,1,1)+P_{29} \Delta F(2,1,1,2)+P_{30} \Delta F(2,1,1,3)+\cdots+P_{52} \Delta F(2,3,3,1) \\
+P_{53} \Delta F(2,3,3,2)+P_{54} \Delta F(2,3,3,3) \\
W_{3}^{1}=F_{3}^{1}+P_{55} \Delta F(3,1,1,1)+P_{56} \Delta F(3,1,1,2)+P_{57} \Delta F(3,1,1,3)+\cdots+P_{79} \Delta F(3,3,3,1) \\
+P_{80} \Delta F(3,3,3,2)+P_{81} \Delta F(3,3,3,3)
\end{gathered}
$$


$W_{1}^{2}=F_{1}^{2}+P_{1} \Delta F(1,1,1,1)+P_{2} \Delta F(1,1,1,2)+P_{3} \Delta F(1,1,1,3)+\cdots+P_{9} \Delta F(1,1,3,3)$

$+P_{28} \Delta F(2,1,1,1)+P_{29} \Delta F(2,1,1,2)+\cdots+P_{36} \Delta F(2,1,3,3)+P_{55} \Delta F(3,1,1,1)$

$+\cdots+P_{62} \Delta F(3,1,3,2)+P_{63} \Delta F(3,1,3,3)$

$W_{2}^{2}=F_{2}^{2}+P_{10} \Delta F(1,2,1,1)+P_{11} \Delta F(1,2,1,2)+P_{12} \Delta F(1,2,1,3)+\cdots+P_{18} \Delta F(1,2,3,3)$

$+P_{37} \Delta F(2,2,1,1)+P_{38} \Delta F(2,2,1,2)+\cdots+P_{45} \Delta F(2,2,3,3)+P_{64} \Delta F(3,2,1,1)$

$+\cdots+P_{71} \Delta F(3,2,3,2)+P_{72} \Delta F(3,2,3,3)$

$W_{3}^{2}=F_{3}^{2}+P_{19} \Delta F(1,3,1,1)+P_{20} \Delta F(1,3,1,2)+P_{21} \Delta F(1,3,1,3)+\cdots+P_{27} \Delta F(1,3,3,3)$

$+P_{46} \Delta F(2,3,1,1)+P_{47} \Delta F(2,3,1,2)+\cdots+P_{54} \Delta F(2,3,3,3)+P_{73} \Delta F(3,3,1,1)$

$+\cdots+P_{80} \Delta F(3,3,3,2)+P_{81} \Delta F(3,3,3,3)$

$W_{1}^{3}=F_{1}^{3}+P_{1} \Delta F(1,1,1,1)+P_{2} \Delta F(1,1,1,2)+P_{3} \Delta F(1,1,1,3)+P_{10} \Delta F(1,2,1,1)$

$+P_{11} \Delta F(1,2,1,2)+P_{12} \Delta F(1,2,1,3)+P_{19} \Delta F(1,3,1,1)+P_{20} \Delta F(1,3,1,2)$

$+P_{21} \Delta F(1,3,1,3)+P_{28} \Delta F(2,1,1,1)+P_{29} \Delta F(2,1,1,2)+P_{30} \Delta F(2,1,1,2)$

$+P_{37} \Delta F(2,2,1,1)+P_{38} \Delta F(2,2,1,2)+P_{39} \Delta F(2,2,1,3)+P_{46} \Delta F(2,3,1,1)$

$+P_{47} \Delta F(2,3,1,2)+P_{48} \Delta F(2,3,1,3)+P_{55} \Delta F(3,1,1,1)+P_{56} \Delta F(3,1,1,2)$

$+P_{57} \Delta F(3,1,1,3)+P_{64} \Delta F(3,2,1,1)+P_{65} \Delta F(3,2,1,2)+P_{66} \Delta F(3,2,1,3)$

$+P_{73} \Delta F(3,3,1,1)+P_{74} \Delta F(3,3,1,2)+P_{75} \Delta F(3,3,1,3)$

$W_{2}^{3}=F_{2}^{3}+P_{4} \Delta F(1,1,2,1)+P_{5} \Delta F(1,1,2,2)+P_{6} \Delta F(1,1,2,3)+P_{13} \Delta F(1,2,2,1)$

$+P_{14} \Delta F(1,2,2,2)+P_{15} \Delta F(1,2,2,3)+P_{22} \Delta F(1,3,2,1)+P_{23} \Delta F(1,3,2,2)$

$+P_{24} \Delta F(1,3,2,3)+P_{31} \Delta F(2,1,2,1)+P_{32} \Delta F(2,1,2,2)+P_{33} \Delta F(2,1,2,2)$

$+P_{40} \Delta F(2,2,2,1)+P_{41} \Delta F(2,2,2,2)+P_{42} \Delta F(2,2,2,3)+P_{49} \Delta F(2,3,2,1)$

$+P_{50} \Delta F(2,3,2,2)+P_{51} \Delta F(2,3,2,3)+P_{58} \Delta F(3,1,2,1)+P_{59} \Delta F(3,1,2,2)$

$+P_{60} \Delta F(3,1,2,3)+P_{67} \Delta F(3,2,2,1)+P_{68} \Delta F(3,2,2,2)+P_{69} \Delta F(3,2,2,3)$

$+P_{76} \Delta F(3,3,2,1)+P_{77} \Delta F(3,3,2,2)+P_{78} \Delta F(3,3,2,3)$

$(10)$

$W_{3}^{3}=F_{3}^{3}+P_{7} \Delta F(1,1,3,1)+P_{8} \Delta F(1,1,3,2)+P_{9} \Delta F(1,1,3,3)+P_{6} \Delta F(1,2,3,1)$

$+P_{17} \Delta F(1,2,3,2)+P_{18} \Delta F(1,2,3,3)+P_{25} \Delta F(1,3,3,1)+P_{26} \Delta F(1,3,3,2)$

$+P_{27} \Delta F(1,3,3,3)+P_{34} \Delta F(2,1,3,1)+P_{35} \Delta F(2,1,3,2)+P_{36} \Delta F(2,1,3,2)$

$+P_{43} \Delta F(2,2,3,1)+P_{44} \Delta F(2,2,3,2)+P_{45} \Delta F(2,2,3,3)+P_{52} \Delta F(2,3,3,1)$

$+P_{53} \Delta F(2,3,3,2)+P_{54} \Delta F(2,3,3,3)+P_{61} \Delta F(3,1,3,1)+P_{62} \Delta F(3,1,3,2)$

$+P_{63} \Delta F(3,1,3,3)+P_{70} \Delta F(3,2,3,1)+P_{71} \Delta F(3,2,3,2)+P_{72} \Delta F(3,2,3,3)$

$+P_{79} \Delta F(3,3,3,1)+P_{80} \Delta F(3,3,3,2)+P_{81} \Delta F(3,3,3,3)$

$W_{1}^{4}=F_{1}^{4}+P_{1} \Delta F(1,1,1,1)+P_{4} \Delta F(1,1,2,1)+P_{7} \Delta F(1,1,3,1)+P_{10} \Delta F(1,2,1,1)$

$+P_{13} \Delta F(1,2,2,1)+P_{16} \Delta F(1,2,3,1)+P_{19} \Delta F(1,3,1,1)+P_{22} \Delta F(1,3,2,1)$

$+P_{25} \Delta F(1,3,3,1)+P_{28} \Delta F(2,1,1,1)+P_{31} \Delta F(2,1,2,1)+P_{34} \Delta F(2,1,3,1)$

$+P_{37} \Delta F(2,2,1,1)+P_{40} \Delta F(2,2,2,1)+P_{43} \Delta F(2,2,3,1)+P_{46} \Delta F(2,3,1,1)$

$+P_{49} \Delta F(2,3,2,1)+P_{52} \Delta F(2,3,3,1)+P_{55} \Delta F(3,1,1,1)+P_{58} \Delta F(3,1,2,1)$

$+P_{61} \Delta F(3,1,3,1)+P_{64} \Delta F(3,2,1,1)+P_{67} \Delta F(3,2,2,1)+P_{70} \Delta F(3,2,3,1)$

$+P_{73} \Delta F(3,3,1,1)+P_{76} \Delta F(3,3,2,1)+P_{79} \Delta F(3,3,3,1)$

$W_{2}^{4}=F_{2}^{4}+P_{2} \Delta F(1,1,1,2)+P_{5} \Delta F(1,1,2,2)+P_{8} \Delta F(1,1,3,2)+P_{11} \Delta F(1,2,1,2)$

$+P_{14} \Delta F(1,2,2,2)+P_{17} \Delta F(1,2,3,2)+P_{20} \Delta F(1,3,1,2)+P_{23} \Delta F(1,3,2,2)$

$+P_{26} \Delta F(1,3,3,2)+P_{29} \Delta F(2,1,1,2)+P_{32} \Delta F(2,1,2,2)+P_{35} \Delta F(2,1,3,2)$

$+P_{38} \Delta F(2,2,1,2)+P_{41} \Delta F(2,2,2,2)+P_{44} \Delta F(2,2,3,2)+P_{47} \Delta F(2,3,1,2)$

$+P_{50} \Delta F(2,3,2,2)+P_{53} \Delta F(2,3,3,2)+P_{56} \Delta F(3,1,1,2)+P_{59} \Delta F(3,1,2,2)$

$+P_{62} \Delta F(3,1,3,2)+P_{65} \Delta F(3,2,1,2)+P_{68} \Delta F(3,2,2,2)+P_{71} \Delta F(3,2,3,2)$

$+P_{74} \Delta F(3,3,1,2)+P_{77} \Delta F(3,3,2,2)+P_{80} \Delta F(3,3,3,2)$

(11)

$P_{3} \Delta F(1,1,1,3)+P_{6} \Delta F(1,1,2,3)+P_{9} \Delta F(1,1,3,3)+P_{12} \Delta F(1,2,1,3)$

$+P_{15} \Delta F(1,2,2,3)+P_{18} \Delta F(1,2,3,3)+P_{21} \Delta F(1,3,1,3)+P_{24} \Delta F(1,3,2,3)$

$+P_{27} \Delta F(1,3,3,3)+P_{30} \Delta F(2,1,1,3)+P_{33} \Delta F(2,1,2,3)+P_{36} \Delta F(2,1,3,3)$

$+P_{39} \Delta F(2,2,1,3)+P_{42} \Delta F(2,2,2,3)+P_{45} \Delta F(2,2,3,3)+P_{48} \Delta F(2,3,1,3)$

$+P_{51} \Delta F(2,3,2,3)+P_{54} \Delta F(2,3,3,3)+P_{57} \Delta F(3,1,1,3)+P_{60} \Delta F(3,1,2,3)$

$+P_{63} \Delta F(3,1,3,3)+P_{66} \Delta F(3,2,1,3)+P_{69} \Delta F(3,2,2,3)+P_{72} \Delta F(3,2,3,3)$

$+P_{75} \Delta F(3,3,1,3)+P_{78} \Delta F(3,3,2,3)+P_{81} \Delta F(3,3,3,3)$ 


$$
\bar{W}_{1}=\left(P_{1}+P_{2}+\cdots+P_{27}\right) W_{1}^{1}+\left(P_{28}+P_{29}+\cdots+P_{54}\right) W_{2}^{1}+\left(P_{55}+P_{56}+\cdots+P_{81}\right) W_{3}^{1}
$$

Let's assume that the proportion of the population following the boxed $i^{\text {th }}$ in the next generation is related to the proportion of the population following the boxed $i^{\text {th }}$ in the current generation according to the rule:

$$
\begin{aligned}
& P_{1}^{\prime}=\frac{P_{1} W_{1}^{1}}{\bar{W}_{1}}, P_{2}^{\prime}=\frac{P_{2} W_{1}^{1}}{\bar{W}_{1}}, \ldots, P_{27}^{\prime}=\frac{P_{27} W_{1}^{1}}{\bar{W}_{1}} \\
& P_{28}^{\prime}=\frac{P_{28} W_{2}^{1}}{\bar{W}_{1}}, P_{29}^{\prime}=\frac{P_{29} W_{2}^{1}}{\bar{W}_{1}}, \ldots, P_{54}^{\prime}=\frac{P_{54} W_{2}^{1}}{\bar{W}_{1}} \\
& P_{55}^{\prime}=\frac{P_{55} W_{3}^{1}}{\bar{W}_{1}}, P_{56}^{\prime}=\frac{P_{56} W_{3}^{1}}{\bar{W}_{1}}, \ldots, P_{81}^{\prime}=\frac{P_{81} W_{3}^{1}}{\bar{W}_{1}}
\end{aligned}
$$

We can rewrite these expressions in the following form:

$$
\begin{aligned}
& P_{1}^{\prime}-P_{1}=\frac{P_{1}\left(W_{1}^{1}-\bar{W}\right)}{\bar{W}_{1}}, P_{2}^{\prime}-P_{2}=\frac{P_{2}\left(W_{1}^{1}-\bar{W}\right)}{\bar{W}_{1}}, \ldots, P_{27}^{\prime}-P_{27}=\frac{P_{27}\left(W_{1}^{1}-\bar{W}\right)}{\bar{W}_{1}} \\
& P_{28}^{\prime}-P_{28}=\frac{P_{28}\left(W_{2}^{1}-\bar{W}\right)}{\bar{W}_{1}}, P_{29}^{\prime}-P_{29}=\frac{P_{29}\left(W_{2}^{1}-\bar{W}\right)}{\bar{W}_{1}}, \ldots, P_{54}^{\prime}-P_{54}=\frac{P_{54}\left(W_{2}^{1}-\bar{W}\right)}{\bar{W}_{1}} \\
& P_{55}^{\prime}-P_{55}=\frac{P_{55}\left(W_{3}^{1}-\bar{W}\right)}{\bar{W}_{1}}, P_{56}^{\prime}-P_{56}=\frac{P_{56}\left(W_{3}^{1}-\bar{W}\right)}{\bar{W}_{1}}, \ldots, P_{81}^{\prime}-P_{81}=\frac{P_{81}\left(W_{3}^{1}-\bar{W}\right)}{\bar{W}_{1}}
\end{aligned}
$$

We assume that small difference is in the strategy frequency from one generation to the next are small:

$$
\begin{aligned}
& \frac{d P_{1}}{d t}=\frac{P_{1}\left(W_{1}^{1}-\bar{W}\right)}{\bar{W}_{1}}, \frac{d P_{2}}{d t}=\frac{P_{2}\left(W_{1}^{1}-\bar{W}\right)}{\bar{W}_{1}}, \ldots, \frac{d P_{27}}{d t}=\frac{P_{27}\left(W_{1}^{1}-\bar{W}\right)}{\bar{W}_{1}} \\
& \frac{d P_{28}}{d t}=\frac{P_{28}\left(W_{2}^{1}-\bar{W}\right)}{\bar{W}_{1}}, \frac{d P_{29}}{d t}=\frac{P_{29}\left(W_{2}^{1}-\bar{W}\right)}{\bar{W}_{1}}, \ldots, \frac{d P_{54}}{d t}=\frac{P_{54}\left(W_{2}^{1}-\bar{W}\right)}{\bar{W}_{1}} \\
& \frac{d P_{55}}{d t}=\frac{P_{55}\left(W_{3}^{1}-\bar{W}\right)}{\bar{W}_{1}}, \frac{d P_{56}}{d t}=\frac{P_{56}\left(W_{3}^{1}-\bar{W}\right)}{\bar{W}_{1}}, \ldots, \frac{d P_{81}}{d t}=\frac{P_{81}\left(W_{3}^{1}-\bar{W}\right)}{\bar{W}_{1}}
\end{aligned}
$$

These equations were to provide continuous dynamics for evolutionary game theory and are known as the replicator dynamics.

\section{Case study}

The proposed method in this paper, as already mentioned, is purposely designed for one of the biggest Food Industries in Iran called 'TAYHOO'.

\subsection{Strategic options of Food Industry supply chain in TAYHOO}

In this section, we define strategic options in perspective of BSC to TAYHOO. In order to avoid being trapped to a high-complicated model, strategic options are limited to three. For this purpose, first areas which suit a set of strategies are designed to help extract a lot of studies and interviews with experts in the company. At this stage, a total of 25 major strategies were identified. In the next stage, the designed questionnaires were bilateral for these prioritized strategies. To this end, 135 questionnaires were distributed and 120 were turned in based on which the analysis is performed. Output results that introduce strategic priorities and the restrictions specified in each field were limited by only three major strategies. Following the analysis of the results, the reliability of the questionnaire was calculated to be 0.73 using Cronbach's alpha formula, indicating that the questionnaire had an acceptable reliability index. Finally, the default original model was introduced based on strategic issues (see Fig. 2). 
Table 3

Result of the questionnaires

\begin{tabular}{|c|c|c|c|c|c|c|c|c|c|}
\hline 금 & Factors & $\begin{array}{c}\text { No. of } \\
\text { Respondent }\end{array}$ & Mean & SD & 믈 & Factors & $\begin{array}{c}\text { No. of } \\
\text { Respondent }\end{array}$ & Mean & SD \\
\hline \multirow{3}{*}{ 烝 } & $\begin{array}{l}\mathrm{F}_{1}: \text { Reduce the } \\
\text { volatility of of } \\
\text { budget }\end{array}$ & 120 & 5.850 & 0.788869 & \multirow{3}{*}{ 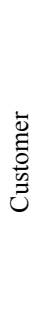 } & $\begin{array}{l}\mathrm{C}_{1} \text { : Develop a plan to } \\
\text { improve the ordering and } \\
\text { distribution Leadtime }\end{array}$ & 120 & 5.758 & 1.493766 \\
\hline & $\begin{array}{c}\mathrm{F}_{2}: \text { Reduce the cost } \\
\text { per hour }\end{array}$ & 120 & 6.508 & 0.70413 & & $\begin{array}{c}\mathrm{C}_{2} \text { :Improving } \\
\text { accountability and } \\
\text { responsibility for returning } \\
\text { faulty goods }\end{array}$ & 120 & 5.550 & 1.442278 \\
\hline & $\begin{array}{l}\mathrm{F}_{3}: \text { Improved sales } \\
\text { than planning }\end{array}$ & 120 & 6.358 & 0.985815 & & $\begin{array}{c}\mathrm{C}_{3} \text { :Improve customer } \\
\text { satisfaction }\end{array}$ & 120 & 5.783 & 1.391731 \\
\hline \multirow{3}{*}{ 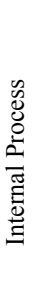 } & $\begin{array}{l}\mathrm{I}_{1}: \text { Reduce planned } \\
\text { process cycle time }\end{array}$ & 120 & 6.275 & 1.317163 & \multirow{3}{*}{ 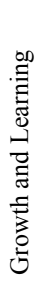 } & $\begin{array}{c}\mathrm{L}_{1}: \text { Make greater use of } \\
\text { technology tools }\end{array}$ & 120 & 4.367 & 1.105459 \\
\hline & $\begin{array}{c}\mathrm{I}_{2}: \text { Reduce purchase } \\
\text { order cycle time }\end{array}$ & 120 & 5.883 & 1.305952 & & $\begin{array}{l}\mathrm{L}_{2} \text { : Practical training and } \\
\text { staff development in order } \\
\text { to distribute tasks }\end{array}$ & 120 & 4.633 & 1.127664 \\
\hline & $\begin{array}{l}\mathrm{I}_{3}: \text { Reduce the } \\
\text { overall cost of } \\
\text { Inventory }\end{array}$ & 120 & 5.708 & 1.255703 & & $\begin{array}{l}\mathrm{L}_{3} \text { : Developing new ideas } \\
\text { to improve }\end{array}$ & 120 & 4.167 & 1.070516 \\
\hline
\end{tabular}

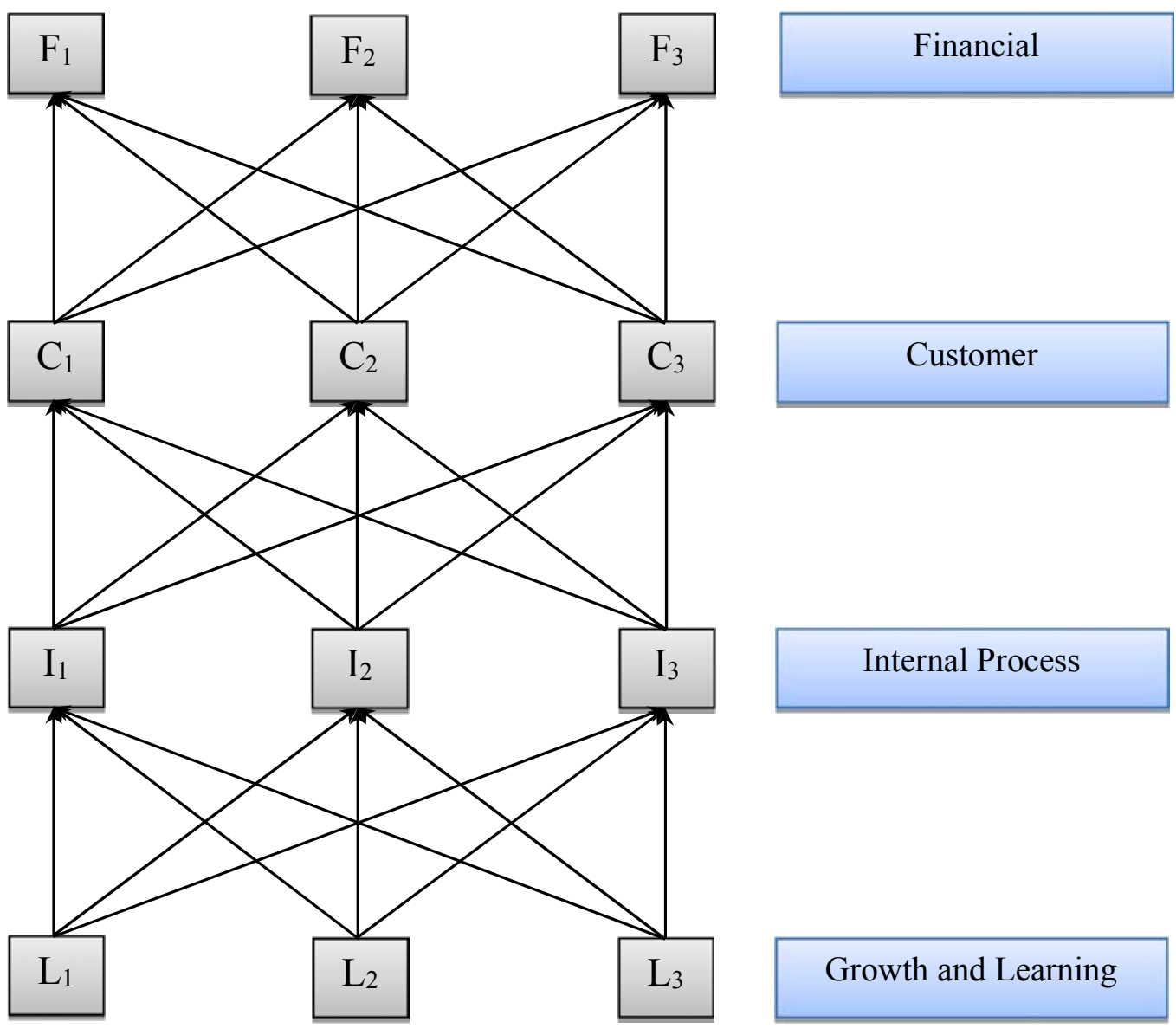

Fig. 2. Primary path analysis model 


\subsection{Determine the relationship between strategies by path analysis}

To begin this step, measurement indicators must have been set for each strategy. Data on these indicators were collected for 12 month period. With this information, we developed the 27 hypothesis tests by twosided correlation $t$ test assuming. These hypothesis tests were accomplished among different components of path analysis based model mutually. Information about these tests have been given in Table 4 .

Table 4

Result of the two-sided correlation $t$ hypothesis test

\begin{tabular}{|c|c|c|c|c|c|c|}
\hline $\begin{array}{l}\text { Relative } \\
\text { Element }\end{array}$ & $\begin{array}{l}\text { Independent } \\
\text { Element }\end{array}$ & Efficacy & Hypothesis & t- Value & $\alpha$ & $\begin{array}{c}\text { Result of } \\
\text { Test }\end{array}$ \\
\hline I1 & L1 & + & $\mathrm{H} 1$ & 3.96 & 0.01 & $\sqrt{ }$ \\
\hline $\mathrm{I} 2$ & L1 & + & $\mathrm{H} 2$ & 4.80 & 0.01 & $\sqrt{ }$ \\
\hline I3 & L1 & + & H3 & 5.78 & 0.01 & $\sqrt{ }$ \\
\hline I1 & $\mathrm{L} 2$ & + & $\mathrm{H} 4$ & 1.45 & - & $\times$ \\
\hline $\mathrm{I} 2$ & L2 & + & H5 & 4.04 & 0.01 & $\sqrt{ }$ \\
\hline I3 & $\mathrm{L} 2$ & + & H6 & 2.18 & 0.05 & $\sqrt{ }$ \\
\hline I1 & L3 & + & $\mathrm{H} 7$ & 1.75 & - & $\times$ \\
\hline $\mathrm{I} 2$ & L3 & + & H8 & 1.17 & - & $\times$ \\
\hline I3 & L3 & + & H9 & 1.88 & 0.10 & $\sqrt{ }$ \\
\hline $\mathrm{C} 1$ & I1 & + & H10 & 3.78 & 0.01 & $\sqrt{ }$ \\
\hline $\mathrm{C} 2$ & I1 & + & H11 & 0.60 & - & $\times$ \\
\hline $\mathrm{C} 3$ & I1 & + & H12 & 0.83 & - & $\times$ \\
\hline $\mathrm{C} 1$ & $\mathrm{I} 2$ & + & H13 & 3.27 & 0.01 & $\sqrt{ }$ \\
\hline $\mathrm{C} 2$ & $\mathrm{I} 2$ & + & $\mathrm{H} 14$ & 2.26 & 0.05 & $\sqrt{ }$ \\
\hline $\mathrm{C} 3$ & $\mathrm{I} 2$ & + & H15 & 3.01 & 0.05 & $\sqrt{ }$ \\
\hline $\mathrm{C} 1$ & $\mathrm{I} 3$ & + & H16 & 3.34 & 0.01 & $\sqrt{ }$ \\
\hline $\mathrm{C} 2$ & I3 & + & H17 & 1.72 & - & $x$ \\
\hline $\mathrm{C} 3$ & I3 & + & H18 & 1.89 & 0.10 & $\sqrt{ }$ \\
\hline $\mathrm{F} 1$ & $\mathrm{C} 1$ & + & H19 & 3.91 & 0.01 & $\sqrt{ }$ \\
\hline $\mathrm{F} 2$ & $\mathrm{C} 1$ & + & $\mathrm{H} 20$ & 5.06 & 0.01 & $\sqrt{ }$ \\
\hline F3 & $\mathrm{C} 1$ & + & H 21 & 0.85 & - & $\times$ \\
\hline $\mathrm{F} 1$ & $\mathrm{C} 2$ & + & $\mathrm{H} 22$ & 1.29 & - & $x$ \\
\hline $\mathrm{F} 2$ & $\mathrm{C} 2$ & + & $\mathrm{H} 23$ & 2.65 & 0.05 & $\sqrt{ }$ \\
\hline F3 & $\mathrm{C} 2$ & + & $\mathrm{H} 24$ & 0.22 & - & $\times$ \\
\hline F1 & C3 & + & $\mathrm{H} 25$ & 1.68 & - & $\times$ \\
\hline $\mathrm{F} 2$ & C3 & + & $\mathrm{H} 26$ & 2.30 & 0.05 & $\sqrt{ }$ \\
\hline F3 & $\mathrm{C} 3$ & + & $\mathrm{H} 27$ & 2.12 & 0.10 & $\sqrt{ }$ \\
\hline
\end{tabular}


The final path analysis model is changed as follows.

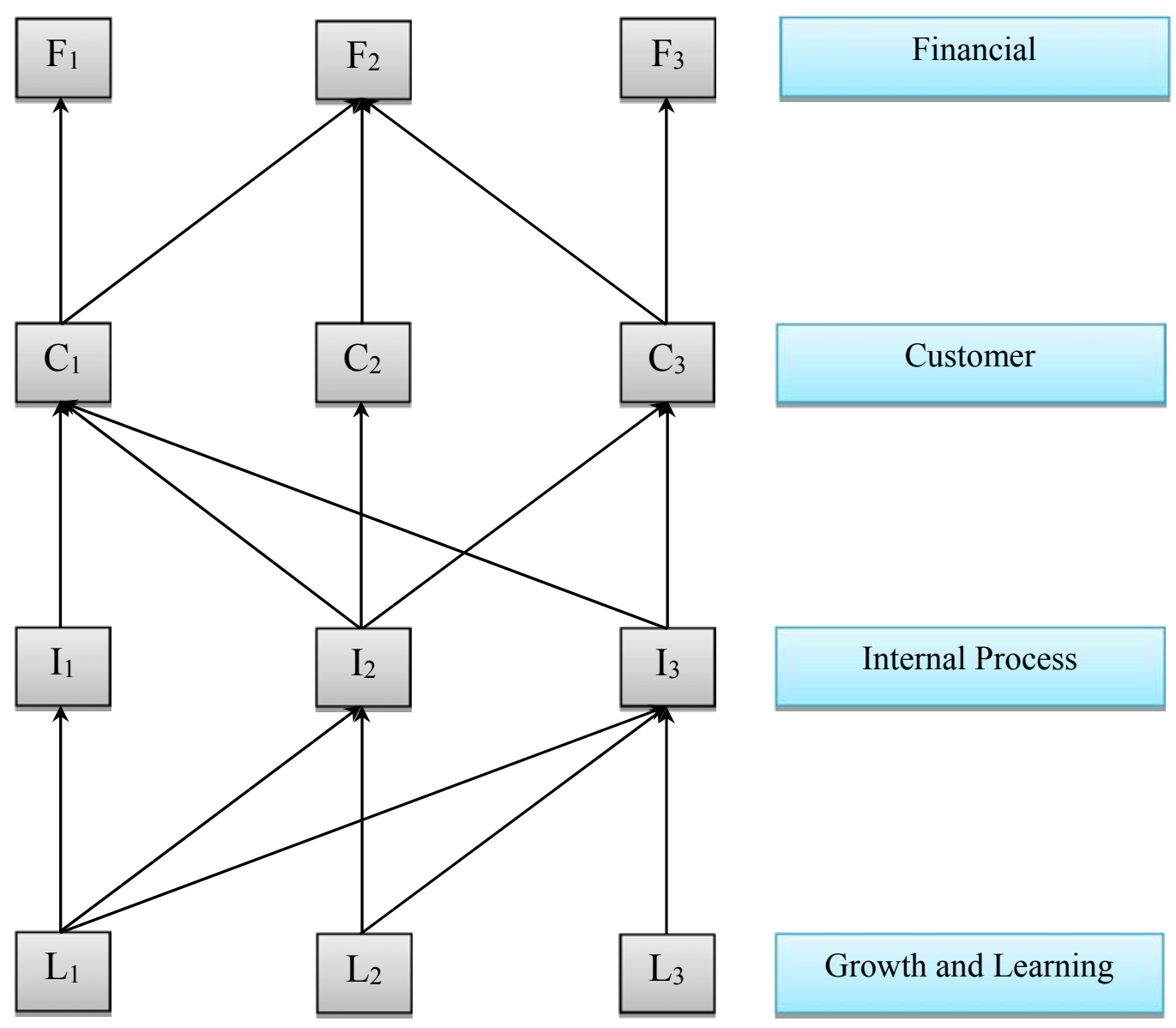

Fig. 3 Final path analysis model

\subsection{Calculation of Shapely Value}

After validating the desired relationships, we calculated Shapely values among the extracted components as a strategy in order for each aspect of balanced scorecard to be considered as a player to determine the effectiveness of components in achieving major goals. In the first, we must determine the initial value of $V(i)$, with $i$ as a component, to determine Shapely values of the components based on the drawn path in the previous section. To determine this value, we used hierarchical analysis approach in fuzzy mode (FAHP). After calculating weights for each component using FAHP method, we will consider these values as $V(i)$. Next, we will consider calculated paths to determine the Shapely value for each component in the path. The Shapely values of 24 drawn paths are show in Table 5.

After determining paths, we were assisted by experts, calculated the necessary values for determining Shapely values. Table 5 presents a detailed specification of the necessary information. After that, based on the rules and proven theorem of Shapely value, we calculated these values for each component on the respective path. 
Table 5

Component of Shapely Value

\begin{tabular}{|c|c|c|c|}
\hline $\mathrm{V}\left(\mathrm{C}_{1}\right)=0.145$ & $\mathrm{~V}\left(\mathrm{I}_{1}\right)=0.047$ & $\mathrm{~V}\left(\mathrm{~L}_{1}\right)=0.035$ & \multirow{5}{*}{$\mathrm{L}_{1} \rightarrow \mathrm{I}_{1} \rightarrow \mathrm{C}_{1} \rightarrow \mathrm{F}_{1}$} \\
\hline $\mathrm{V}\left(\mathrm{L}_{1}, \mathrm{C}_{1}\right)=0.210$ & $\mathrm{~V}\left(\mathrm{~L}_{1}, \mathrm{I}_{1}\right)=0.116$ & $\mathrm{~V}\left(\mathrm{~F}_{1}\right)=0.145$ & \\
\hline $\mathrm{V}\left(\mathrm{I}_{1}, \mathrm{~F}_{1}\right)=0.238$ & $\mathrm{~V}\left(\mathrm{I}_{1}, \mathrm{C}_{1}\right)=0.272$ & $\mathrm{~V}\left(\mathrm{~L}_{1}, \mathrm{~F}_{1}\right)=0.180$ & \\
\hline $\mathrm{V}\left(\mathrm{L}_{1}, \mathrm{I}_{1}, \mathrm{~F}_{1}\right)=0.455$ & $\mathrm{~V}\left(\mathrm{~L}_{1}, \mathrm{I}_{1}, \mathrm{C}_{1}\right)=0.321$ & $\mathrm{~V}\left(\mathrm{C}_{1}, \mathrm{~F}_{1}\right)=0.290$ & \\
\hline $\mathrm{V}\left(\mathrm{L}_{1}, \mathrm{I}_{1}, \mathrm{C}_{1}, \mathrm{~F}_{1}\right)=1$ & $\mathrm{~V}\left(\mathrm{I}_{1}, \mathrm{C}_{1}, \mathrm{~F}_{1}\right)=0.606$ & $\mathrm{~V}\left(\mathrm{~L}_{1}, \mathrm{C}_{1}, \mathrm{~F}_{1}\right)=0.475$ & \\
\hline$V\left(C_{1}\right)=0.145$ & $\mathrm{~V}\left(\mathrm{I}_{1}\right)=0.047$ & $\mathrm{~V}\left(\mathrm{~L}_{1}\right)=0.035$ & \multirow{5}{*}{$\mathrm{L}_{1} \rightarrow \mathrm{I}_{1} \rightarrow \mathrm{C}_{1} \rightarrow \mathrm{F}_{2}$} \\
\hline $\mathrm{V}\left(\mathrm{L}_{1}, \mathrm{C}_{1}\right)=0.210$ & $\mathrm{~V}\left(\mathrm{~L}_{1}, \mathrm{I}_{1}\right)=0.116$ & $\mathrm{~V}\left(\mathrm{~F}_{2}\right)=0.201$ & \\
\hline $\mathrm{V}\left(\mathrm{I}_{1}, \mathrm{~F}_{2}\right)=0.350$ & $\mathrm{~V}\left(\mathrm{I}_{1}, \mathrm{C}_{1}\right)=0.272$ & $\mathrm{~V}\left(\mathrm{~L}_{1}, \mathrm{~F}_{2}\right)=0.311$ & \\
\hline $\mathrm{V}\left(\mathrm{L}_{1}, \mathrm{I}_{1}, \mathrm{~F}_{2}\right)=0.566$ & $\mathrm{~V}\left(\mathrm{~L}_{1}, \mathrm{I}_{1}, \mathrm{C}_{1}\right)=0.321$ & $\mathrm{~V}\left(\mathrm{C}_{1}, \mathrm{~F}_{2}\right)=0.378$ & \\
\hline $\mathrm{V}\left(\mathrm{L}_{1}, \mathrm{I}_{1}, \mathrm{C}_{1}, \mathrm{~F}_{2}\right)=1$ & $\mathrm{~V}\left(\mathrm{I}_{1}, \mathrm{C}_{1}, \mathrm{~F}_{2}\right)=0.589$ & $\mathrm{~V}\left(\mathrm{~L}_{1}, \mathrm{C}_{1}, \mathrm{~F}_{2}\right)=0.571$ & \\
\hline $\mathrm{V}\left(\mathrm{C}_{1}\right)=0.145$ & $\mathrm{~V}\left(\mathrm{I}_{2}\right)=0.066$ & $\mathrm{~V}\left(\mathrm{~L}_{1}\right)=0.035$ & \multirow{5}{*}{$\mathrm{L}_{1} \rightarrow \mathrm{I}_{2} \rightarrow \mathrm{C}_{1} \rightarrow \mathrm{F}_{1}$} \\
\hline $\mathrm{V}\left(\mathrm{L}_{1}, \mathrm{C}_{1}\right)=0.210$ & $\mathrm{~V}\left(\mathrm{~L}_{1}, \mathrm{I}_{2}\right)=0.121$ & $\mathrm{~V}\left(\mathrm{~F}_{1}\right)=0.145$ & \\
\hline $\mathrm{V}\left(\mathrm{I}_{2}, \mathrm{~F}_{1}\right)=0.298$ & $\mathrm{~V}\left(\mathrm{I}_{2}, \mathrm{C}_{1}\right)=0.232$ & $\mathrm{~V}\left(\mathrm{~L}_{1}, \mathrm{~F}_{1}\right)=0.180$ & \\
\hline $\mathrm{V}\left(\mathrm{L}_{1}, \mathrm{I}_{2}, \mathrm{~F}_{1}\right)=0.491$ & $\mathrm{~V}\left(\mathrm{~L}_{1}, \mathrm{I}_{2}, \mathrm{C}_{1}\right)=0.378$ & $\mathrm{~V}\left(\mathrm{C}_{1}, \mathrm{~F}_{1}\right)=0.290$ & \\
\hline $\mathrm{V}\left(\mathrm{L}_{1}, \mathrm{I}_{2}, \mathrm{C}_{1}, \mathrm{~F}_{1}\right)=1$ & $\mathrm{~V}\left(\mathrm{I}_{2}, \mathrm{C}_{1}, \mathrm{~F}_{1}\right)=0.534$ & $\mathrm{~V}\left(\mathrm{~L}_{1}, \mathrm{C}_{1}, \mathrm{~F}_{1}\right)=0.475$ & \\
\hline $\mathrm{V}\left(\mathrm{C}_{1}\right)=0.145$ & $\mathrm{~V}\left(\mathrm{I}_{2}\right)=0.066$ & $\mathrm{~V}\left(\mathrm{~L}_{1}\right)=0.035$ & \multirow{5}{*}{$\mathrm{L}_{1} \rightarrow \mathrm{I}_{2} \rightarrow \mathrm{C}_{1} \rightarrow \mathrm{F}_{2}$} \\
\hline $\mathrm{V}\left(\mathrm{L}_{1}, \mathrm{C}_{1}\right)=0.210$ & $\mathrm{~V}\left(\mathrm{~L}_{1}, \mathrm{I}_{2}\right)=0.121$ & $\mathrm{~V}\left(\mathrm{~F}_{2}\right)=0.201$ & \\
\hline $\mathrm{V}\left(\mathrm{I}_{2}, \mathrm{~F}_{2}\right)=0.277$ & $\mathrm{~V}\left(\mathrm{I}_{2}, \mathrm{C}_{1}\right)=0.232$ & $\mathrm{~V}\left(\mathrm{~L}_{1}, \mathrm{~F}_{2}\right)=0.311$ & \\
\hline $\mathrm{V}\left(\mathrm{L}_{1}, \mathrm{I}_{2}, \mathrm{~F}_{2}\right)=0.603$ & $\mathrm{~V}\left(\mathrm{~L}_{1}, \mathrm{I}_{2}, \mathrm{C}_{1}\right)=0.378$ & $\mathrm{~V}\left(\mathrm{C}_{1}, \mathrm{~F}_{2}\right)=0.378$ & \\
\hline $\mathrm{V}\left(\mathrm{L}_{1}, \mathrm{I}_{2}, \mathrm{C}_{1}, \mathrm{~F}_{2}\right)=1$ & $\mathrm{~V}\left(\mathrm{I}_{2}, \mathrm{C}_{1}, \mathrm{~F}_{2}\right)=0.679$ & $\mathrm{~V}\left(\mathrm{~L}_{1}, \mathrm{C}_{1}, \mathrm{~F}_{2}\right)=0.571$ & \\
\hline $\mathrm{V}\left(\mathrm{C}_{2}\right)=0.091$ & $\mathrm{~V}\left(\mathrm{I}_{2}\right)=0.066$ & $\mathrm{~V}\left(\mathrm{~L}_{1}\right)=0.035$ & \multirow{5}{*}{$\mathrm{L}_{1} \rightarrow \mathrm{I}_{2} \rightarrow \mathrm{C}_{2} \rightarrow \mathrm{F}_{2}$} \\
\hline $\mathrm{V}\left(\mathrm{L}_{1}, \mathrm{C}_{2}\right)=0.126$ & $\mathrm{~V}\left(\mathrm{~L}_{1}, \mathrm{I}_{2}\right)=0.121$ & $\mathrm{~V}\left(\mathrm{~F}_{2}\right)=0.201$ & \\
\hline $\mathrm{V}\left(\mathrm{I}_{2}, \mathrm{~F}_{2}\right)=0.277$ & $\mathrm{~V}\left(\mathrm{I}_{2}, \mathrm{C}_{2}\right)=0.157$ & $\mathrm{~V}\left(\mathrm{~L}_{1}, \mathrm{~F}_{2}\right)=0.311$ & \\
\hline $\mathrm{V}\left(\mathrm{L}_{1}, \mathrm{I}_{2}, \mathrm{~F}_{2}\right)=0.603$ & $\mathrm{~V}\left(\mathrm{~L}_{1}, \mathrm{I}_{2}, \mathrm{C}_{2}\right)=0.272$ & $\mathrm{~V}\left(\mathrm{C}_{2}, \mathrm{~F}_{2}\right)=0.301$ & \\
\hline $\mathrm{V}\left(\mathrm{L}_{1}, \mathrm{I}_{2}, \mathrm{C}_{2}, \mathrm{~F}_{2}\right)=1$ & $\mathrm{~V}\left(\mathrm{I}_{2}, \mathrm{C}_{2}, \mathrm{~F}_{2}\right)=0.501$ & $\mathrm{~V}\left(\mathrm{~L}_{1}, \mathrm{C}_{2}, \mathrm{~F}_{2}\right)=0.462$ & \\
\hline $\mathrm{V}\left(\mathrm{C}_{3}\right)=0.062$ & $\mathrm{~V}\left(\mathrm{I}_{2}\right)=0.066$ & $\mathrm{~V}\left(\mathrm{~L}_{1}\right)=0.035$ & \multirow{5}{*}{$\mathrm{L}_{1} \rightarrow \mathrm{I}_{2} \rightarrow \mathrm{C}_{3} \rightarrow \mathrm{F}_{2}$} \\
\hline $\mathrm{V}\left(\mathrm{L}_{1}, \mathrm{C}_{3}\right)=0.097$ & $\mathrm{~V}\left(\mathrm{~L}_{1}, \mathrm{I}_{2}\right)=0.121$ & $\mathrm{~V}\left(\mathrm{~F}_{2}\right)=0.201$ & \\
\hline $\mathrm{V}\left(\mathrm{I}_{2}, \mathrm{~F}_{2}\right)=0.277$ & $\mathrm{~V}\left(\mathrm{I}_{2}, \mathrm{C}_{3}\right)=0.181$ & $\mathrm{~V}\left(\mathrm{~L}_{1}, \mathrm{~F}_{2}\right)=0.311$ & \\
\hline $\mathrm{V}\left(\mathrm{L}_{1}, \mathrm{I}_{2}, \mathrm{~F}_{2}\right)=0.603$ & $\mathrm{~V}\left(\mathrm{~L}_{1}, \mathrm{I}_{2}, \mathrm{C}_{3}\right)=0.266$ & $\mathrm{~V}\left(\mathrm{C}_{3}, \mathrm{~F}_{2}\right)=0.275$ & \\
\hline $\mathrm{V}\left(\mathrm{L}_{1}, \mathrm{I}_{2}, \mathrm{C}_{3}, \mathrm{~F}_{2}\right)=1$ & $\mathrm{~V}\left(\mathrm{I}_{2}, \mathrm{C}_{3}, \mathrm{~F}_{2}\right)=0.427$ & $\mathrm{~V}\left(\mathrm{~L}_{1}, \mathrm{C}_{3}, \mathrm{~F}_{2}\right)=0.421$ & \\
\hline $\mathrm{V}\left(\mathrm{C}_{3}\right)=0.062$ & $\mathrm{~V}\left(\mathrm{I}_{2}\right)=0.066$ & $\mathrm{~V}\left(\mathrm{~L}_{1}\right)=0.035$ & \multirow{5}{*}{$\mathrm{L}_{1} \rightarrow \mathrm{I}_{2} \rightarrow \mathrm{C}_{3} \rightarrow \mathrm{F}_{3}$} \\
\hline $\mathrm{V}\left(\mathrm{L}_{1}, \mathrm{C}_{3}\right)=0.097$ & $\mathrm{~V}\left(\mathrm{~L}_{1}, \mathrm{I}_{2}\right)=0.121$ & $\mathrm{~V}\left(\mathrm{~F}_{3}\right)=0.149$ & \\
\hline $\mathrm{V}\left(\mathrm{I}_{2}, \mathrm{~F}_{3}\right)=0.235$ & $\mathrm{~V}\left(\mathrm{I}_{2}, \mathrm{C}_{3}\right)=0.181$ & $\mathrm{~V}\left(\mathrm{~L}_{1}, \mathrm{~F}_{3}\right)=0.201$ & \\
\hline $\mathrm{V}\left(\mathrm{L}_{1}, \mathrm{I}_{2}, \mathrm{~F}_{3}\right)=0.499$ & $\mathrm{~V}\left(\mathrm{~L}_{1}, \mathrm{I}_{2}, \mathrm{C}_{3}\right)=0.266$ & $\mathrm{~V}\left(\mathrm{C}_{3}, \mathrm{~F}_{3}\right)=0.240$ & \\
\hline $\mathrm{V}\left(\mathrm{L}_{1}, \mathrm{I}_{2}, \mathrm{C}_{3}, \mathrm{~F}_{3}\right)=1$ & $\mathrm{~V}\left(\mathrm{I}_{2}, \mathrm{C}_{3}, \mathrm{~F}_{3}\right)=0.360$ & $\mathrm{~V}\left(\mathrm{~L}_{1}, \mathrm{C}_{3}, \mathrm{~F}_{3}\right)=0.369$ & \\
\hline $\mathrm{V}\left(\mathrm{C}_{1}\right)=0.145$ & $\mathrm{~V}\left(\mathrm{I}_{3}\right)=0.021$ & $\mathrm{~V}\left(\mathrm{~L}_{1}\right)=0.035$ & \multirow{5}{*}{$\mathrm{L}_{1} \rightarrow \mathrm{I}_{3} \rightarrow \mathrm{C}_{1} \rightarrow \mathrm{F}_{1}$} \\
\hline $\mathrm{V}\left(\mathrm{L}_{1}, \mathrm{C}_{1}\right)=0.210$ & $\mathrm{~V}\left(\mathrm{~L}_{1}, \mathrm{I}_{3}\right)=0.066$ & $\mathrm{~V}\left(\mathrm{~F}_{1}\right)=0.145$ & \\
\hline $\mathrm{V}\left(\mathrm{I}_{3}, \mathrm{~F}_{1}\right)=0.175$ & $\mathrm{~V}\left(\mathrm{I}_{3}, \mathrm{C}_{1}\right)=0.168$ & $\mathrm{~V}\left(\mathrm{~L}_{1}, \mathrm{~F}_{1}\right)=0.180$ & \\
\hline $\mathrm{V}\left(\mathrm{L}_{1}, \mathrm{I}_{3}, \mathrm{~F}_{1}\right)=0.346$ & $\mathrm{~V}\left(\mathrm{~L}_{1}, \mathrm{I}_{3}, \mathrm{C}_{1}\right)=0.301$ & $\mathrm{~V}\left(\mathrm{C}_{1}, \mathrm{~F}_{1}\right)=0.290$ & \\
\hline $\mathrm{V}\left(\mathrm{L}_{1}, \mathrm{I}_{3}, \mathrm{C}_{1}, \mathrm{~F}_{1}\right)=1$ & $\mathrm{~V}\left(\mathrm{I}_{3}, \mathrm{C}_{1}, \mathrm{~F}_{1}\right)=0.312$ & $\mathrm{~V}\left(\mathrm{~L}_{1}, \mathrm{C}_{1}, \mathrm{~F}_{1}\right)=0.475$ & \\
\hline $\mathrm{V}\left(\mathrm{C}_{1}\right)=0.145$ & $\mathrm{~V}\left(\mathrm{I}_{3}\right)=0.021$ & $\mathrm{~V}\left(\mathrm{~L}_{1}\right)=0.035$ & \multirow{5}{*}{$\mathrm{L}_{1} \rightarrow \mathrm{I}_{3} \rightarrow \mathrm{C}_{1} \rightarrow \mathrm{F}_{2}$} \\
\hline $\mathrm{V}\left(\mathrm{L}_{1}, \mathrm{C}_{1}\right)=0.210$ & $\mathrm{~V}\left(\mathrm{~L}_{1}, \mathrm{I}_{3}\right)=0.066$ & $\mathrm{~V}\left(\mathrm{~F}_{2}\right)=0.201$ & \\
\hline $\mathrm{V}\left(\mathrm{I}_{3}, \mathrm{~F}_{2}\right)=0.272$ & $\mathrm{~V}\left(\mathrm{I}_{3}, \mathrm{C}_{1}\right)=0.168$ & $\mathrm{~V}\left(\mathrm{~L}_{1}, \mathrm{~F}_{2}\right)=0.311$ & \\
\hline $\mathrm{V}\left(\mathrm{L}_{1}, \mathrm{I}_{3}, \mathrm{~F}_{2}\right)=0.458$ & $\mathrm{~V}\left(\mathrm{~L}_{1}, \mathrm{I}_{3}, \mathrm{C}_{1}\right)=0.301$ & $\mathrm{~V}\left(\mathrm{C}_{1}, \mathrm{~F}_{2}\right)=0.378$ & \\
\hline $\mathrm{V}\left(\mathrm{L}_{1}, \mathrm{I}_{3}, \mathrm{C}_{1}, \mathrm{~F}_{2}\right)=1$ & $\mathrm{~V}\left(\mathrm{I}_{3}, \mathrm{C}_{1}, \mathrm{~F}_{2}\right)=0.513$ & $\mathrm{~V}\left(\mathrm{~L}_{1}, \mathrm{C}_{1}, \mathrm{~F}_{2}\right)=0.571$ & \\
\hline $\mathrm{V}\left(\mathrm{C}_{3}\right)=0.062$ & $\mathrm{~V}\left(\mathrm{I}_{3}\right)=0.021$ & $\mathrm{~V}\left(\mathrm{~L}_{1}\right)=0.035$ & \multirow{5}{*}{$\mathrm{L}_{1} \rightarrow \mathrm{I}_{3} \rightarrow \mathrm{C}_{3} \rightarrow \mathrm{F}_{2}$} \\
\hline $\mathrm{V}\left(\mathrm{L}_{1}, \mathrm{C}_{3}\right)=0.097$ & $\mathrm{~V}\left(\mathrm{~L}_{1}, \mathrm{I}_{3}\right)=0.066$ & $\mathrm{~V}\left(\mathrm{~F}_{2}\right)=0.201$ & \\
\hline $\mathrm{V}\left(\mathrm{I}_{3}, \mathrm{~F}_{2}\right)=0.272$ & $\mathrm{~V}\left(\mathrm{I}_{3}, \mathrm{C}_{3}\right)=0.085$ & $\mathrm{~V}\left(\mathrm{~L}_{1}, \mathrm{~F}_{2}\right)=0.311$ & \\
\hline $\mathrm{V}\left(\mathrm{L}_{1}, \mathrm{I}_{3}, \mathrm{~F}_{2}\right)=0.458$ & $\mathrm{~V}\left(\mathrm{~L}_{1}, \mathrm{I}_{3}, \mathrm{C}_{3}\right)=0.242$ & $\mathrm{~V}\left(\mathrm{C}_{3}, \mathrm{~F}_{2}\right)=0.275$ & \\
\hline $\mathrm{V}\left(\mathrm{L}_{1}, \mathrm{I}_{3}, \mathrm{C}_{3}, \mathrm{~F}_{2}\right)=1$ & $\mathrm{~V}\left(\mathrm{I}_{3}, \mathrm{C}_{3}, \mathrm{~F}_{2}\right)=0.426$ & $\mathrm{~V}\left(\mathrm{~L}_{1}, \mathrm{C}_{3}, \mathrm{~F}_{2}\right)=0.421$ & \\
\hline $\mathrm{V}\left(\mathrm{C}_{3}\right)=0.062$ & $\mathrm{~V}\left(\mathrm{I}_{3}\right)=0.021$ & $\mathrm{~V}\left(\mathrm{~L}_{1}\right)=0.035$ & \multirow{5}{*}{$\mathrm{L}_{1} \rightarrow \mathrm{I}_{3} \rightarrow \mathrm{C}_{3} \rightarrow \mathrm{F}_{3}$} \\
\hline $\mathrm{V}\left(\mathrm{L}_{1}, \mathrm{C}_{3}\right)=0.097$ & $\mathrm{~V}\left(\mathrm{~L}_{1}, \mathrm{I}_{3}\right)=0.066$ & $\mathrm{~V}\left(\mathrm{~F}_{3}\right)=0.149$ & \\
\hline $\mathrm{V}\left(\mathrm{I}_{3}, \mathrm{~F}_{3}\right)=0.170$ & $\mathrm{~V}\left(\mathrm{I}_{3}, \mathrm{C}_{3}\right)=0.085$ & $\mathrm{~V}\left(\mathrm{~L}_{1}, \mathrm{~F}_{3}\right)=0.201$ & \\
\hline $\mathrm{V}\left(\mathrm{L}_{1}, \mathrm{I}_{3}, \mathrm{~F}_{3}\right)=0.354$ & $\mathrm{~V}\left(\mathrm{~L}_{1}, \mathrm{I}_{3}, \mathrm{C}_{3}\right)=0.242$ & $\mathrm{~V}\left(\mathrm{C}_{3}, \mathrm{~F}_{3}\right)=0.240$ & \\
\hline $\mathrm{V}\left(\mathrm{L}_{1}, \mathrm{I}_{3}, \mathrm{C}_{3}, \mathrm{~F}_{3}\right)=1$ & $\mathrm{~V}\left(\mathrm{I}_{3}, \mathrm{C}_{3}, \mathrm{~F}_{3}\right)=0.348$ & $\mathrm{~V}\left(\mathrm{~L}_{1}, \mathrm{C}_{3}, \mathrm{~F}_{3}\right)=0.369$ & \\
\hline $\mathrm{V}\left(\mathrm{C}_{1}\right)=0.145$ & $\mathrm{~V}\left(\mathrm{I}_{2}\right)=0.066$ & $\mathrm{~V}\left(\mathrm{~L}_{2}\right)=0.013$ & \multirow{5}{*}{$\mathrm{L}_{2} \rightarrow \mathrm{I}_{2} \rightarrow \mathrm{C}_{1} \rightarrow \mathrm{F}_{1}$} \\
\hline $\mathrm{V}\left(\mathrm{L}_{2}, \mathrm{C}_{1}\right)=0.223$ & $\mathrm{~V}\left(\mathrm{~L}_{2}, \mathrm{I}_{2}\right)=0.080$ & $\mathrm{~V}\left(\mathrm{~F}_{1}\right)=0.145$ & \\
\hline $\mathrm{V}\left(\mathrm{I}_{2}, \mathrm{~F}_{1}\right)=0.298$ & $\mathrm{~V}\left(\mathrm{I}_{2}, \mathrm{C}_{1}\right)=0.232$ & $\mathrm{~V}\left(\mathrm{~L}_{2}, \mathrm{~F}_{1}\right)=0.158$ & \\
\hline $\mathrm{V}\left(\mathrm{L}_{2}, \mathrm{I}_{2}, \mathrm{~F}_{1}\right)=0.403$ & $\mathrm{~V}\left(\mathrm{~L}_{2}, \mathrm{I}_{2}, \mathrm{C}_{1}\right)=0.386$ & $\mathrm{~V}\left(\mathrm{C}_{1}, \mathrm{~F}_{1}\right)=0.290$ & \\
\hline $\mathrm{V}\left(\mathrm{L}_{2}, \mathrm{I}_{2}, \mathrm{C}_{1}, \mathrm{~F}_{1}\right)=1$ & $\mathrm{~V}\left(\mathrm{I}_{2}, \mathrm{C}_{1}, \mathrm{~F}_{1}\right)=0.534$ & $\mathrm{~V}\left(\mathrm{~L}_{2}, \mathrm{C}_{1}, \mathrm{~F}_{1}\right)=0.428$ & \\
\hline
\end{tabular}


Table 5

Component of Shapely Value-continue

\begin{tabular}{|c|c|c|c|}
\hline $\mathrm{V}\left(\mathrm{C}_{1}\right)=0.145$ & $\mathrm{~V}\left(\mathrm{I}_{2}\right)=0.066$ & $\mathrm{~V}\left(\mathrm{~L}_{2}\right)=0.013$ & \multirow{5}{*}{$\mathrm{L}_{2} \rightarrow \mathrm{I}_{2} \rightarrow \mathrm{C}_{1} \rightarrow \mathrm{F}_{2}$} \\
\hline $\mathrm{V}\left(\mathrm{L}_{2}, \mathrm{C}_{1}\right)=0.223$ & $\mathrm{~V}\left(\mathrm{~L}_{2}, \mathrm{I}_{2}\right)=0.080$ & $\mathrm{~V}\left(\mathrm{~F}_{2}\right)=0.201$ & \\
\hline $\mathrm{V}\left(\mathrm{I}_{2}, \mathrm{~F}_{2}\right)=0.277$ & $\mathrm{~V}\left(\mathrm{I}_{2}, \mathrm{C}_{1}\right)=0.232$ & $\mathrm{~V}\left(\mathrm{~L}_{2}, \mathrm{~F}_{2}\right)=0.216$ & \\
\hline $\mathrm{V}\left(\mathrm{L}_{2}, \mathrm{I}_{2}, \mathrm{~F}_{2}\right)=0.503$ & $\mathrm{~V}\left(\mathrm{~L}_{2}, \mathrm{I}_{2}, \mathrm{C}_{1}\right)=0.386$ & $\mathrm{~V}\left(\mathrm{C}_{1}, \mathrm{~F}_{2}\right)=0.378$ & \\
\hline $\mathrm{V}\left(\mathrm{L}_{2}, \mathrm{I}_{2}, \mathrm{C}_{1}, \mathrm{~F}_{2}\right)=1$ & $\mathrm{~V}\left(\mathrm{I}_{2}, \mathrm{C}_{1}, \mathrm{~F}_{2}\right)=0.534$ & $\mathrm{~V}\left(\mathrm{~L}_{2}, \mathrm{C}_{1}, \mathrm{~F}_{2}\right)=0.538$ & \\
\hline $\mathrm{V}\left(\mathrm{C}_{2}\right)=0.091$ & $\mathrm{~V}\left(\mathrm{I}_{2}\right)=0.066$ & $\mathrm{~V}\left(\mathrm{~L}_{2}\right)=0.013$ & \multirow{5}{*}{$\mathrm{L}_{2} \rightarrow \mathrm{I}_{2} \rightarrow \mathrm{C}_{2} \rightarrow \mathrm{F}_{2}$} \\
\hline $\mathrm{V}\left(\mathrm{L}_{2}, \mathrm{C}_{2}\right)=0.138$ & $\mathrm{~V}\left(\mathrm{~L}_{2}, \mathrm{I}_{2}\right)=0.080$ & $\mathrm{~V}\left(\mathrm{~F}_{2}\right)=0.201$ & \\
\hline $\mathrm{V}\left(\mathrm{I}_{2}, \mathrm{~F}_{2}\right)=0.277$ & $\mathrm{~V}\left(\mathrm{I}_{2}, \mathrm{C}_{2}\right)=0.157$ & $\mathrm{~V}\left(\mathrm{~L}_{2}, \mathrm{~F}_{2}\right)=0.216$ & \\
\hline $\mathrm{V}\left(\mathrm{L}_{2}, \mathrm{I}_{2}, \mathrm{~F}_{2}\right)=0.503$ & $\mathrm{~V}\left(\mathrm{~L}_{2}, \mathrm{I}_{2}, \mathrm{C}_{2}\right)=0.340$ & $\mathrm{~V}\left(\mathrm{C}_{2}, \mathrm{~F}_{2}\right)=0.301$ & \\
\hline $\mathrm{V}\left(\mathrm{L}_{2}, \mathrm{I}_{2}, \mathrm{C}_{2}, \mathrm{~F}_{2}\right)=1$ & $\mathrm{~V}\left(\mathrm{I}_{2}, \mathrm{C}_{2}, \mathrm{~F}_{2}\right)=0.501$ & $\mathrm{~V}\left(\mathrm{~L}_{2}, \mathrm{C}_{2}, \mathrm{~F}_{2}\right)=0.431$ & \\
\hline $\mathrm{V}\left(\mathrm{C}_{3}\right)=0.062$ & $\mathrm{~V}\left(\mathrm{I}_{2}\right)=0.066$ & $\mathrm{~V}\left(\mathrm{~L}_{2}\right)=0.013$ & \multirow{5}{*}{$\mathrm{L}_{2} \rightarrow \mathrm{I}_{2} \rightarrow \mathrm{C}_{3} \rightarrow \mathrm{F}_{2}$} \\
\hline $\mathrm{V}\left(\mathrm{L}_{2}, \mathrm{C}_{3}\right)=0.075$ & $\mathrm{~V}\left(\mathrm{~L}_{2}, \mathrm{I}_{2}\right)=0.080$ & $\mathrm{~V}\left(\mathrm{~F}_{2}\right)=0.201$ & \\
\hline $\mathrm{V}\left(\mathrm{I}_{2}, \mathrm{~F}_{2}\right)=0.277$ & $\mathrm{~V}\left(\mathrm{I}_{2}, \mathrm{C}_{3}\right)=0.181$ & $\mathrm{~V}\left(\mathrm{~L}_{2}, \mathrm{~F}_{2}\right)=0.216$ & \\
\hline $\mathrm{V}\left(\mathrm{L}_{2}, \mathrm{I}_{2}, \mathrm{~F}_{2}\right)=0.503$ & $\mathrm{~V}\left(\mathrm{~L}_{2}, \mathrm{I}_{2}, \mathrm{C}_{3}\right)=0.211$ & $\mathrm{~V}\left(\mathrm{C}_{3}, \mathrm{~F}_{2}\right)=0.275$ & \\
\hline $\mathrm{V}\left(\mathrm{L}_{2}, \mathrm{I}_{2}, \mathrm{C}_{3}, \mathrm{~F}_{2}\right)=1$ & $\mathrm{~V}\left(\mathrm{I}_{2}, \mathrm{C}_{3}, \mathrm{~F}_{2}\right)=0.427$ & $\mathrm{~V}\left(\mathrm{~L}_{2}, \mathrm{C}_{3}, \mathrm{~F}_{2}\right)=0.390$ & \\
\hline $\mathrm{V}\left(\mathrm{C}_{3}\right)=0.062$ & $\mathrm{~V}\left(\mathrm{I}_{2}\right)=0.066$ & $\mathrm{~V}\left(\mathrm{~L}_{2}\right)=0.013$ & \multirow{5}{*}{$\mathrm{L}_{2} \rightarrow \mathrm{I}_{2} \rightarrow \mathrm{C}_{3} \rightarrow \mathrm{F}_{3}$} \\
\hline $\mathrm{V}\left(\mathrm{L}_{2}, \mathrm{C}_{3}\right)=0.075$ & $\mathrm{~V}\left(\mathrm{~L}_{2}, \mathrm{I}_{2}\right)=0.080$ & $\mathrm{~V}\left(\mathrm{~F}_{3}\right)=0.149$ & \\
\hline $\mathrm{V}\left(\mathrm{I}_{2}, \mathrm{~F}_{3}\right)=0.235$ & $\mathrm{~V}\left(\mathrm{I}_{2}, \mathrm{C}_{3}\right)=0.181$ & $\mathrm{~V}\left(\mathrm{~L}_{2}, \mathrm{~F}_{3}\right)=0.185$ & \\
\hline $\mathrm{V}\left(\mathrm{L}_{2}, \mathrm{I}_{2}, \mathrm{~F}_{3}\right)=0.385$ & $\mathrm{~V}\left(\mathrm{~L}_{2}, \mathrm{I}_{2}, \mathrm{C}_{3}\right)=0.211$ & $\mathrm{~V}\left(\mathrm{C}_{3}, \mathrm{~F}_{3}\right)=0.240$ & \\
\hline $\mathrm{V}\left(\mathrm{L}_{2}, \mathrm{I}_{2}, \mathrm{C}_{3}, \mathrm{~F}_{3}\right)=1$ & $\mathrm{~V}\left(\mathrm{I}_{2}, \mathrm{C}_{3}, \mathrm{~F}_{3}\right)=0.360$ & $\mathrm{~V}\left(\mathrm{~L}_{2}, \mathrm{C}_{3}, \mathrm{~F}_{3}\right)=0.316$ & \\
\hline $\mathrm{V}\left(\mathrm{C}_{1}\right)=0.145$ & $\mathrm{~V}\left(\mathrm{I}_{3}\right)=0.021$ & $\mathrm{~V}\left(\mathrm{~L}_{2}\right)=0.013$ & \multirow{5}{*}{$\mathrm{L}_{2} \rightarrow \mathrm{I}_{3} \rightarrow \mathrm{C}_{1} \rightarrow \mathrm{F}_{1}$} \\
\hline $\mathrm{V}\left(\mathrm{L}_{2}, \mathrm{C}_{1}\right)=0.223$ & $\mathrm{~V}\left(\mathrm{~L}_{2}, \mathrm{I}_{3}\right)=0.048$ & $\mathrm{~V}\left(\mathrm{~F}_{1}\right)=0.145$ & \\
\hline $\mathrm{V}\left(\mathrm{I}_{3}, \mathrm{~F}_{1}\right)=0.175$ & $\mathrm{~V}\left(\mathrm{I}_{3}, \mathrm{C}_{1}\right)=0.168$ & $\mathrm{~V}\left(\mathrm{~L}_{2}, \mathrm{~F}_{1}\right)=0.158$ & \\
\hline $\mathrm{V}\left(\mathrm{L}_{2}, \mathrm{I}_{3}, \mathrm{~F}_{1}\right)=0.308$ & $\mathrm{~V}\left(\mathrm{~L}_{2}, \mathrm{I}_{3}, \mathrm{C}_{1}\right)=0.358$ & $\mathrm{~V}\left(\mathrm{C}_{1}, \mathrm{~F}_{1}\right)=0.290$ & \\
\hline $\mathrm{V}\left(\mathrm{L}_{2}, \mathrm{I}_{3}, \mathrm{C}_{1}, \mathrm{~F}_{1}\right)=1$ & $\mathrm{~V}\left(\mathrm{I}_{3}, \mathrm{C}_{1}, \mathrm{~F}_{1}\right)=0.312$ & $\mathrm{~V}\left(\mathrm{~L}_{2}, \mathrm{C}_{1}, \mathrm{~F}_{1}\right)=0.428$ & \\
\hline $\mathrm{V}\left(\mathrm{C}_{1}\right)=0.145$ & $\mathrm{~V}\left(\mathrm{I}_{3}\right)=0.021$ & $\mathrm{~V}\left(\mathrm{~L}_{2}\right)=0.013$ & \multirow{5}{*}{$\mathrm{L}_{2} \rightarrow \mathrm{I}_{3} \rightarrow \mathrm{C}_{1} \rightarrow \mathrm{F}_{2}$} \\
\hline $\mathrm{V}\left(\mathrm{L}_{2}, \mathrm{C}_{1}\right)=0.223$ & $\mathrm{~V}\left(\mathrm{~L}_{2}, \mathrm{I}_{3}\right)=0.048$ & $\mathrm{~V}\left(\mathrm{~F}_{2}\right)=0.201$ & \\
\hline $\mathrm{V}\left(\mathrm{I}_{3}, \mathrm{~F}_{2}\right)=0.272$ & $\mathrm{~V}\left(\mathrm{I}_{3}, \mathrm{C}_{1}\right)=0.168$ & $\mathrm{~V}\left(\mathrm{~L}_{2}, \mathrm{~F}_{2}\right)=0.216$ & \\
\hline $\mathrm{V}\left(\mathrm{L}_{2}, \mathrm{I}_{3}, \mathrm{~F}_{2}\right)=0.470$ & $\mathrm{~V}\left(\mathrm{~L}_{2}, \mathrm{I}_{3}, \mathrm{C}_{1}\right)=0.358$ & $\mathrm{~V}\left(\mathrm{C}_{1}, \mathrm{~F}_{2}\right)=0.378$ & \\
\hline $\mathrm{V}\left(\mathrm{L}_{2}, \mathrm{I}_{3}, \mathrm{C}_{1}, \mathrm{~F}_{2}\right)=1$ & $\mathrm{~V}\left(\mathrm{I}_{3}, \mathrm{C}_{1}, \mathrm{~F}_{2}\right)=0.513$ & $\mathrm{~V}\left(\mathrm{~L}_{2}, \mathrm{C}_{1}, \mathrm{~F}_{2}\right)=0.538$ & \\
\hline $\mathrm{V}\left(\mathrm{C}_{3}\right)=0.062$ & $\mathrm{~V}\left(\mathrm{I}_{3}\right)=0.021$ & $\mathrm{~V}\left(\mathrm{~L}_{2}\right)=0.013$ & \multirow{5}{*}{$\mathrm{L}_{2} \rightarrow \mathrm{I}_{3} \rightarrow \mathrm{C}_{3} \rightarrow \mathrm{F}_{2}$} \\
\hline $\mathrm{V}\left(\mathrm{L}_{2}, \mathrm{C}_{3}\right)=0.075$ & $\mathrm{~V}\left(\mathrm{~L}_{2}, \mathrm{I}_{3}\right)=0.048$ & $\mathrm{~V}\left(\mathrm{~F}_{2}\right)=0.201$ & \\
\hline $\mathrm{V}\left(\mathrm{I}_{3}, \mathrm{~F}_{2}\right)=0.272$ & $\mathrm{~V}\left(\mathrm{I}_{3}, \mathrm{C}_{3}\right)=0.085$ & $\mathrm{~V}\left(\mathrm{~L}_{2}, \mathrm{~F}_{2}\right)=0.216$ & \\
\hline $\mathrm{V}\left(\mathrm{L}_{2}, \mathrm{I}_{3}, \mathrm{~F}_{2}\right)=0.470$ & $\mathrm{~V}\left(\mathrm{~L}_{2}, \mathrm{I}_{3}, \mathrm{C}_{3}\right)=0.234$ & $\mathrm{~V}\left(\mathrm{C}_{3}, \mathrm{~F}_{2}\right)=0.275$ & \\
\hline $\mathrm{V}\left(\mathrm{L}_{2}, \mathrm{I}_{3}, \mathrm{C}_{3}, \mathrm{~F}_{2}\right)=1$ & $\mathrm{~V}\left(\mathrm{I}_{3}, \mathrm{C}_{3}, \mathrm{~F}_{2}\right)=0.426$ & $\mathrm{~V}\left(\mathrm{~L}_{2}, \mathrm{C}_{3}, \mathrm{~F}_{2}\right)=0.390$ & \\
\hline $\mathrm{V}\left(\mathrm{C}_{3}\right)=0.062$ & $\mathrm{~V}\left(\mathrm{I}_{3}\right)=0.021$ & $\mathrm{~V}\left(\mathrm{~L}_{2}\right)=0.013$ & \multirow{5}{*}{$\mathrm{L}_{2} \rightarrow \mathrm{I}_{3} \rightarrow \mathrm{C}_{3} \rightarrow \mathrm{F}_{3}$} \\
\hline $\mathrm{V}\left(\mathrm{L}_{2}, \mathrm{C}_{3}\right)=0.075$ & $\mathrm{~V}\left(\mathrm{~L}_{2}, \mathrm{I}_{3}\right)=0.048$ & $\mathrm{~V}\left(\mathrm{~F}_{3}\right)=0.149$ & \\
\hline $\mathrm{V}\left(\mathrm{I}_{3}, \mathrm{~F}_{3}\right)=0.170$ & $\mathrm{~V}\left(\mathrm{I}_{3}, \mathrm{C}_{3}\right)=0.085$ & $\mathrm{~V}\left(\mathrm{~L}_{2}, \mathrm{~F}_{3}\right)=0.185$ & \\
\hline $\mathrm{V}\left(\mathrm{L}_{2}, \mathrm{I}_{3}, \mathrm{~F}_{3}\right)=0.289$ & $\mathrm{~V}\left(\mathrm{~L}_{2}, \mathrm{I}_{3}, \mathrm{C}_{3}\right)=0.234$ & $\mathrm{~V}\left(\mathrm{C}_{3}, \mathrm{~F}_{3}\right)=0.240$ & \\
\hline $\mathrm{V}\left(\mathrm{L}_{2}, \mathrm{I}_{3}, \mathrm{C}_{3}, \mathrm{~F}_{3}\right)=1$ & $\mathrm{~V}\left(\mathrm{I}_{3}, \mathrm{C}_{3}, \mathrm{~F}_{3}\right)=0.348$ & $\mathrm{~V}\left(\mathrm{~L}_{2}, \mathrm{C}_{3}, \mathrm{~F}_{3}\right)=0.316$ & \\
\hline $\mathrm{V}\left(\mathrm{C}_{1}\right)=0.145$ & $\mathrm{~V}\left(\mathrm{I}_{3}\right)=0.021$ & $\mathrm{~V}\left(\mathrm{~L}_{3}\right)=0.025$ & \multirow{5}{*}{$\mathrm{L}_{3} \rightarrow \mathrm{I}_{3} \rightarrow \mathrm{C}_{1} \rightarrow \mathrm{F}_{1}$} \\
\hline $\mathrm{V}\left(\mathrm{L}_{3}, \mathrm{C}_{1}\right)=0.170$ & $\mathrm{~V}\left(\mathrm{~L}_{3}, \mathrm{I}_{3}\right)=0.046$ & $\mathrm{~V}\left(\mathrm{~F}_{1}\right)=0.145$ & \\
\hline $\mathrm{V}\left(\mathrm{I}_{3}, \mathrm{~F}_{1}\right)=0.175$ & $\mathrm{~V}\left(\mathrm{I}_{3}, \mathrm{C}_{1}\right)=0.168$ & $\mathrm{~V}\left(\mathrm{~L}_{3}, \mathrm{~F}_{1}\right)=0.170$ & \\
\hline $\mathrm{V}\left(\mathrm{L}_{3}, \mathrm{I}_{3}, \mathrm{~F}_{1}\right)=0.324$ & $\mathrm{~V}\left(\mathrm{~L}_{3}, \mathrm{I}_{3}, \mathrm{C}_{1}\right)=0.286$ & $\mathrm{~V}\left(\mathrm{C}_{1}, \mathrm{~F}_{1}\right)=0.290$ & \\
\hline $\mathrm{V}\left(\mathrm{L}_{3}, \mathrm{I}_{3}, \mathrm{C}_{1}, \mathrm{~F}_{1}\right)=1$ & $\mathrm{~V}\left(\mathrm{I}_{3}, \mathrm{C}_{1}, \mathrm{~F}_{1}\right)=0.312$ & $\mathrm{~V}\left(\mathrm{~L}_{3}, \mathrm{C}_{1}, \mathrm{~F}_{1}\right)=0.473$ & \\
\hline $\mathrm{V}\left(\mathrm{C}_{1}\right)=0.145$ & $\mathrm{~V}\left(\mathrm{I}_{3}\right)=0.021$ & $\mathrm{~V}\left(\mathrm{~L}_{3}\right)=0.025$ & \multirow{5}{*}{$\mathrm{L}_{3} \rightarrow \mathrm{I}_{3} \rightarrow \mathrm{C}_{1} \rightarrow \mathrm{F}_{2}$} \\
\hline $\mathrm{V}\left(\mathrm{L}_{3}, \mathrm{C}_{1}\right)=0.170$ & $\mathrm{~V}\left(\mathrm{~L}_{3}, \mathrm{I}_{3}\right)=0.046$ & $\mathrm{~V}\left(\mathrm{~F}_{2}\right)=0.201$ & \\
\hline $\mathrm{V}\left(\mathrm{I}_{3}, \mathrm{~F}_{2}\right)=0.272$ & $\mathrm{~V}\left(\mathrm{I}_{3}, \mathrm{C}_{1}\right)=0.168$ & $\mathrm{~V}\left(\mathrm{~L}_{3}, \mathrm{~F}_{2}\right)=0.225$ & \\
\hline $\mathrm{V}\left(\mathrm{L}_{3}, \mathrm{I}_{3}, \mathrm{~F}_{2}\right)=0.419$ & $\mathrm{~V}\left(\mathrm{~L}_{3}, \mathrm{I}_{3}, \mathrm{C}_{1}\right)=0.286$ & $\mathrm{~V}\left(\mathrm{C}_{1}, \mathrm{~F}_{2}\right)=0.378$ & \\
\hline $\mathrm{V}\left(\mathrm{L}_{3}, \mathrm{I}_{3}, \mathrm{C}_{1}, \mathrm{~F}_{2}\right)=1$ & $\mathrm{~V}\left(\mathrm{I}_{3}, \mathrm{C}_{1}, \mathrm{~F}_{2}\right)=0.513$ & $\mathrm{~V}\left(\mathrm{~L}_{3}, \mathrm{C}_{1}, \mathrm{~F}_{2}\right)=0.524$ & \\
\hline $\mathrm{V}\left(\mathrm{C}_{3}\right)=0.062$ & $\mathrm{~V}\left(\mathrm{I}_{3}\right)=0.021$ & $\mathrm{~V}\left(\mathrm{~L}_{3}\right)=0.025$ & \multirow{5}{*}{$\mathrm{L}_{3} \rightarrow \mathrm{I}_{3} \rightarrow \mathrm{C}_{3} \rightarrow \mathrm{F}_{2}$} \\
\hline $\mathrm{V}\left(\mathrm{L}_{3}, \mathrm{C}_{3}\right)=0.092$ & $\mathrm{~V}\left(\mathrm{~L}_{3}, \mathrm{I}_{3}\right)=0.046$ & $\mathrm{~V}\left(\mathrm{~F}_{2}\right)=0.201$ & \\
\hline $\mathrm{V}\left(\mathrm{I}_{3}, \mathrm{~F}_{2}\right)=0.272$ & $\mathrm{~V}\left(\mathrm{I}_{3}, \mathrm{C}_{3}\right)=0.085$ & $\mathrm{~V}\left(\mathrm{~L}_{3}, \mathrm{~F}_{2}\right)=0.225$ & \\
\hline $\mathrm{V}\left(\mathrm{L}_{3}, \mathrm{I}_{3}, \mathrm{~F}_{2}\right)=0.419$ & $\mathrm{~V}\left(\mathrm{~L}_{3}, \mathrm{I}_{3}, \mathrm{C}_{3}\right)=0.233$ & $\mathrm{~V}\left(\mathrm{C}_{3}, \mathrm{~F}_{2}\right)=0.275$ & \\
\hline $\mathrm{V}\left(\mathrm{L}_{3}, \mathrm{I}_{3}, \mathrm{C}_{3}, \mathrm{~F}_{2}\right)=1$ & $\mathrm{~V}\left(\mathrm{I}_{3}, \mathrm{C}_{3}, \mathrm{~F}_{2}\right)=0.426$ & $\mathrm{~V}\left(\mathrm{~L}_{3}, \mathrm{C}_{3}, \mathrm{~F}_{2}\right)=0.407$ & \\
\hline $\mathrm{V}\left(\mathrm{C}_{3}\right)=0.062$ & $\mathrm{~V}\left(\mathrm{I}_{3}\right)=0.021$ & $\mathrm{~V}\left(\mathrm{~L}_{3}\right)=0.025$ & \multirow{5}{*}{$\mathrm{L}_{3} \rightarrow \mathrm{I}_{3} \rightarrow \mathrm{C}_{3} \rightarrow \mathrm{F}_{3}$} \\
\hline $\mathrm{V}\left(\mathrm{L}_{3}, \mathrm{C}_{3}\right)=0.092$ & $\mathrm{~V}\left(\mathrm{~L}_{3}, \mathrm{I}_{3}\right)=0.046$ & $\mathrm{~V}\left(\mathrm{~F}_{3}\right)=0.149$ & \\
\hline $\mathrm{V}\left(\mathrm{I}_{3}, \mathrm{~F}_{3}\right)=0.170$ & $\mathrm{~V}\left(\mathrm{I}_{3}, \mathrm{C}_{3}\right)=0.085$ & $\mathrm{~V}\left(\mathrm{~L}_{3}, \mathrm{~F}_{3}\right)=0.175$ & \\
\hline $\mathrm{V}\left(\mathrm{L}_{3}, \mathrm{I}_{3}, \mathrm{~F}_{3}\right)=0.331$ & $\mathrm{~V}\left(\mathrm{~L}_{3}, \mathrm{I}_{3}, \mathrm{C}_{3}\right)=0.233$ & $\mathrm{~V}\left(\mathrm{C}_{3}, \mathrm{~F}_{3}\right)=0.240$ & \\
\hline $\mathrm{V}\left(\mathrm{L}_{3}, \mathrm{I}_{3}, \mathrm{C}_{3}, \mathrm{~F}_{3}\right)=1$ & $\mathrm{~V}\left(\mathrm{I}_{3}, \mathrm{C}_{3}, \mathrm{~F}_{3}\right)=0.348$ & $\mathrm{~V}\left(\mathrm{~L}_{3}, \mathrm{C}_{3}, \mathrm{~F}_{3}\right)=0.354$ & \\
\hline
\end{tabular}


Regarding the steps taken, Latin square is designed as shown in Table. Table 3 illustrates a game where finance is appointed as the first mover, the customer is the follower, and internal processes, and growth and learning are assumed to have moved with the first choice. Since the game theory of dynamic behavior does not have rationality in a traditional mode of insight, in a state of evolutionary game theory it can be vacuum filled due to the ambiguity. These cases were encountered in this article that considered the evolutionary game theory and modeling problem with this theory in form of the concept of Nash equation in the dynamic equilibrium approach. Nash equation resulted in the convergence of all players in the long run and will remain stable. By using the information obtained in the previous step Shapely Value of each player in each path can be achieved. These values are entered in the Latin square design.

\section{Table 6}

Final Latin square model

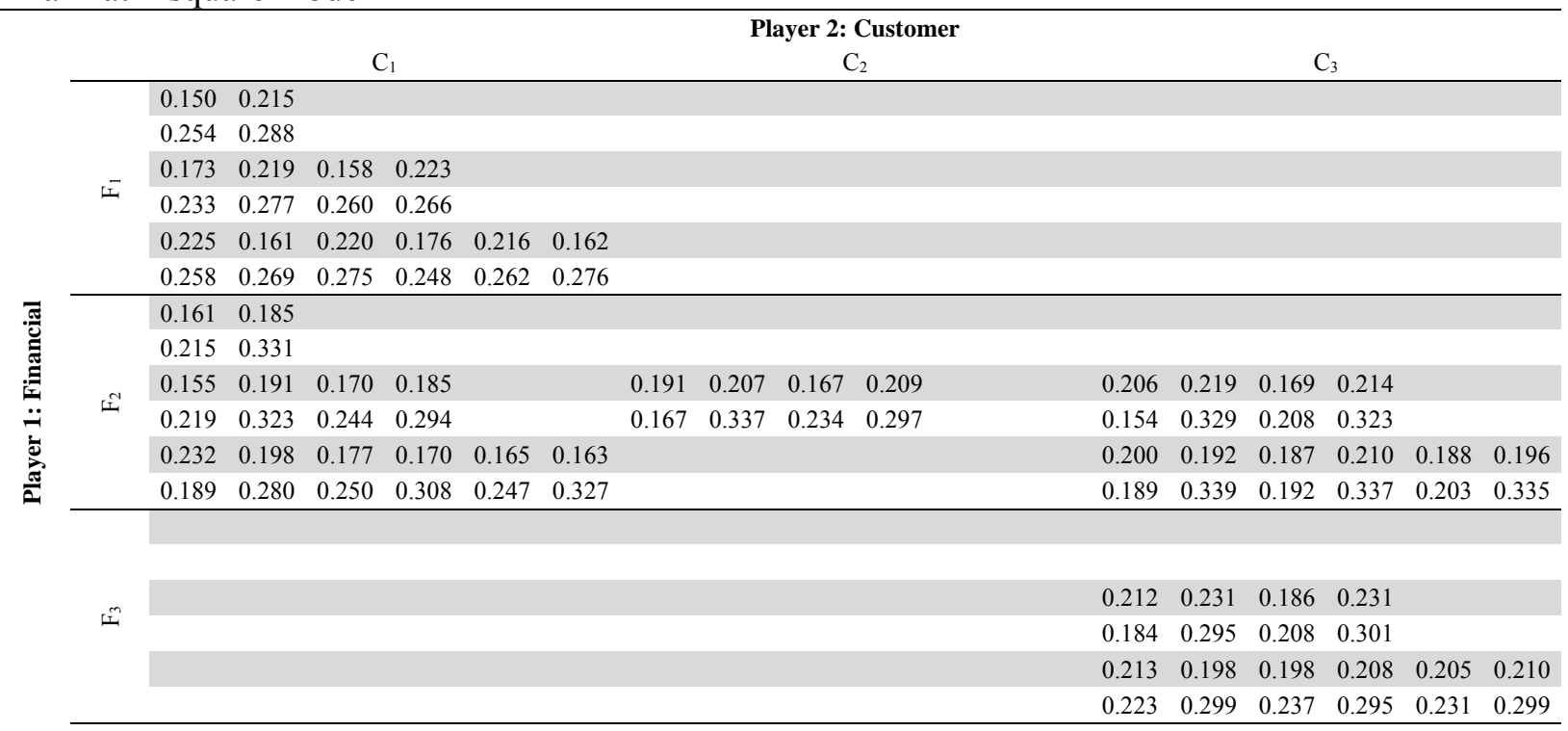

Values that were missing in Latin square design are the paths that were eliminated in the path analysis step. Therefore, they are considered to be zero. This intelligent system database provides the organization that facilitates decision making and shows how the units interact. Suppose TAYHOO has problems in the area of financial supply chain and needs to identify the best combination of strategies to achieve the best results. The combined relationship help us in game theory and we may obtain the most appropriate way to reach the face bring this problem to obtain. The equations for this problem are explained below. Values of $\Delta \mathrm{F}\left(\mathrm{s}_{1}, \mathrm{~s}_{2}, \mathrm{~s}_{3}, \mathrm{~s}_{4}\right)$ are the Shapely Values that are listed in the Latin square design. Initial values of $\mathrm{P}_{\mathrm{i}}$ for boxes that have value are considered to $1 / 24$, and for remain boxes this value is equal to zero.

(we assumption that $\mathrm{F}_{\mathrm{i}} \mathrm{j}=0$; for any $\mathrm{i}=1,2,3, \mathrm{j}=1,2,3,4$ )

$$
\begin{gathered}
W_{1}^{1}=0.288 P_{1}+0.277 P_{4}+0.266 P_{5}+0.269 P_{7}+0.248 P_{8}+0.276 P_{9} \\
W_{2}^{1}=0.331 P_{28}+0.323 P_{31}+0.294 P_{32}+0.280 P_{34}+0.308 P_{35}+0.327 P_{36}+0.337 P_{40} \\
+0.297 P_{41}+0.329 P_{49}+0.323 P_{50}+0.339 P_{52}+0.337 P_{53}+0.335 P_{54} \\
W_{3}^{1}=0.295 P_{76}+0.301 P_{77}+0.299 P_{79}+0.295 P_{80}+0.299 P_{81} \\
\bar{W}_{1}=\left(P_{1}+P_{4}+P_{5}+P_{7}+P_{8}+P_{9}\right) W_{1}^{1} \\
+\left(P_{28}+P_{31}+P_{32}+P_{34}+P_{35}+P_{36}+P_{40}+P_{41}+P_{49}+P_{50}+P_{52}\right. \\
\left.+P_{53}+P_{54}\right) W_{2}^{1}+\left(P_{76}+P_{77}+P_{79}+P_{80}+P_{81}\right) W_{3}^{1}
\end{gathered}
$$

Other equations are computed using Eqs. (6)-(14). 
Value of $P_{i}^{\prime}$ can be obtained by using Eqs. (16-18) for the next generation. The optimal strategy for each player is determined by repeating this calculation. The calculations are coded by computer software. The result of this calculation for TAYHOO company showed that the optimal strategy was the second strategy for the first player. Also, first strategy for the second player, the third strategy for the third player and the first strategy for the fourth plyer are optimal strategies. This means that the optimal box in Latin square design is box 34 . Therefore, $\mathrm{L}_{1} \rightarrow \mathrm{I}_{3} \rightarrow \mathrm{C}_{1} \rightarrow \mathrm{F}_{2}$ is the optimal strategic path for TAYHOO company.

\section{Conclusion}

Performance measurement is an essential element of effective planning and control as well as decision making. Although implementations of performance measures in companies are now widespread. In this study, an innovative method has been presented for performance measurement of food supply chain. In this method, we have used a combination of balanced scorecard, path analysis, cooperative game theory and evolutionary game theory to obtain the best combination of strategic. We have proposed the application of this new approach of combining game theory, path analysis and BSC for food supply chain to TAYHOO with the objective to evaluate their day-to-day business performance in order to select the best strategy in emergency situations. After determining strategies in each aspect of BSC, we determine cause and effect relationships between the strategies defined. Then the best strategic path were determined by using cooperative game theory and evolutionary game theory.

\section{References}

Abu Turab Rizvi, S. (2007). Aumann's and Schelling's game theory: the Nobel Prize in Economic Science, 2005. Review of Political Economy, 19(3), 297-316.

Bhagwat, R., \& Sharma, M. K. (2007). Performance measurement of supply chain management: A balanced scorecard approach. Computers \& Industrial Engineering, 53(1), 43-62.

Bigliardi, B., \& Bottani, E. (2010). Performance measurement in the food supply chain: a balanced scorecard approach. Facilities, 28(5/6), 249-260.

Burney, L. L., Henle, C. A., \& Widener, S. K. (2009). A path model examining the relations among strategic performance measurement system characteristics, organizational justice, and extra-and inrole performance. Accounting, Organizations and Society, 34(3), 305-321.

Cardemil-Katuranic, G., \& Shadbolt, N. (2006). The Balanced Scorecard as a spontaneous framework in an agricultural hybrid cooperative under strategic change: A case study in the New Zealand kiwifruit industry. In World Food and Agribusiness Congress, Buenos Aires, Argentina.

Cebeci, U. (2009). Fuzzy AHP-based decision support system for selecting ERP systems in textile industry by using balanced scorecard. Expert Systems with Applications, 36(5), 8900-8909.

Chia, A., Goh, M., \& Hum, S. H. (2009). Performance measurement in supply chain entities: balanced scorecard perspective. Benchmarking: An International Journal, 16(5), 605-620.

Cho, D. W., Lee, Y. H., Ahn, S. H., \& Hwang, M. K. (2012). A framework for measuring the performance of service supply chain management. Computers \& Industrial Engineering, 62(3), 801-818.

Gilles, R. P. (2010). The cooperative game theory of networks and Hierarchies (Vol. 44). Springer.

Gumbus, A. (2005). Introducing the balanced scorecard: creating metrics to measure performance. Journal of Management Education, 29(4), 617-630.

Gunasekaran, A., \& Kobu, B. (2007). Performance measures and metrics in logistics and supply chain management: a review of recent literature (1995-2004) for research and applications. International Journal of Production Research, 45(12), 2819-2840.

Gunasekaran, A., Patel, C., \& McGaughey, R. E. (2004). A framework for supply chain performance measurement. International Journal of Production Economics, 87(3), 333-347.

Huang, C. F., \& Hsueh, S. L. (2007). A Study on the relationship between intellectual capital and business performance in the engineering consulting industry: A path analysis. Journal of Civil Engineering and Management, 13(4), 265-271. 
Jafari, M., Tootooni, M., Shahanaghi, K., \& Eskandari, M. J. (2013). Determining the relationship between science and technology development indicators in private sector industries of Iran via path analysis based on cooperative game theory. Life Science Journal, 10(3).

Jalali Naini, S. G., Aliahmadi, A. R., \& Jafari-Eskandari, M. (2011). Designing a mixed performance measurement system for environmental supply chain management using evolutionary game theory and balanced scorecard: a case study of an auto industry supply chain. Resources, Conservation and Recycling, 55(6), 593-603.

Kanji, G. K. (2002). Performance measurement system. Total Quality Management, 13(5), 715-728.

Kanji, G. K., \& e Sá, P. M. (2002). Kanji's business scorecard. Total Quality Management, 13(1), 13-27.

Kaplan, R. S., \& Norton, D. P. (1992). The Balanced Scorecard œ Measures That Drive Performance, in: Harvard Business Review, January-February 1992.

Kaplan, R.S., \& Norton, D.P. (1996). Using the Balancesd scorecard as a strategic management system. Harvard Business Review, 74(1), 75-85.

Keegan, D. P., Eiler, R. G., \& Jones, C. R. (1989). Are your performance measures obsolete?. Management accounting, 70(12), 45-50.

Kohlberg, E., \& Mertens, J. F. (1986). On the strategic stability of equilibria. Econometrica: Journal of the Econometric Society, 1003-1037.

Kreps, D. M., \& Wilson, R. (1982). Reputation and imperfect information. Journal of Economic Theory, 27(2), 253-279.

Kuhn, H. W., \& Tucker, A. W. (1958). John von Neumann's work in the theory of games and mathematical economics. Bulletin of the American Mathematical Society, 64(3, Part 2), 100-122.

Lee, A. H., Chen, W. C., \& Chang, C. J. (2008). A fuzzy AHP and BSC approach for evaluating performance of IT department in the manufacturing industry in Taiwan. Expert Systems with Applications, 34(1), 96-107.

Nash Jr, J. F. (1953). Equilibrium points in N-person games. Proceedings of the National Academy of Sciences of the USA, Vol. 36, pp. 48=49. Two-Person Cooperative Games, Econometrica, 21, 128140.

Nash, J. (1951). Non-cooperative games. Annals of Mathematics, 286-295.

Nash, J. F. (1950). Equilibrium points in n-person games. Proceedings of the national academy of sciences, 36(1), 48-49.

Razmi, J., Ghaderi, S. F., Zairi, M., \& Keyno, H. S. (2008). Compiling and prioritizing strategies for electrical energy production from fossil fuel with support of benchmarking. Benchmarking: An International Journal, 15(6), 794-804.

Shaw, S., Grant, D. B., \& Mangan, J. (2010). Developing environmental supply chain performance measures. Benchmarking: An International Journal, 17(3), 320-339.

Tijs, S. H. (2003). Introduction to game theory.

Yang, M. C., \& Tung, Y. C. (2006). Using path analysis to examine causal relationships among balanced scorecard performance indicators for general hospitals: The case of a public hospital system in Taiwan. Health Care Management Review, 31(4), 280-288. 